Databases, Revenues, \& Repertory: The French Stage Online, 16801793 • Données, recettes \& répertoire: La scène en ligne (1680-1793)

\title{
La Comédie-Française par les chiffres, 1752-2020
}

Jeffrey S. Ravel

Published on: Oct 07, 2020

DOI: $10.21428 / 671 \mathrm{~d} 579 \mathrm{e} .1 \mathrm{f} 312 \mathrm{ff} 3$

License: Creative Commons Attribution 4.0 International License (CC-BY 4.0). 


\section{Introduction}

Le Projet des Registres de la Comédie-Française ( $\underline{\mathrm{RCF}}$ ) participe d'un mouvement contemporain où chercheurs en sciences humaines et informaticiens unissent leur force. Ces collaborations, que l'on subsume souvent sous le terme d'humanités numériques, ont donné lieu à des débats passionnés. Leurs détracteurs estiment qu'elles constituent une attaque néo-libérale contre l'université et la recherche avec pour seul dessein de les rendre les plus rentables possible, au détriment de la diversité de l'éducation et des valeurs propres aux sciences humaines $\underline{1}$. Ils affirment que les départements universitaires traditionnels sont de moins en moins financés et que les modèles de carrière établis sont mis en péril, au profit de technologues opposés aux sciences humaines, dont le travail ouvre la voie à des évaluations essentiellement quantitatives. Un article du Chronicle of Higher Education paru à l'automne 2017 est allé jusqu'à défendre l'idée qu'après dix ans, on pouvait désormais avoir la certitude que les humanités numériques étaient un « fiasco ». Son auteur affirme qu'en dépit d'investissements se comptant en millions de dollars et provenant d'universités et de fondations aussi bien publiques que privées, les projets dans ce domaine n'ont presque rien changé aux paradigmes fondamentaux du savoir dans le champ des sciences humaines $\underline{2}$.

Les chercheurs en humanités numériques et ceux qui les défendent ont été prompts à répondre, soutenant que ceux qui les attaquent sont des traditionalistes qui refusent d'acquérir de nouvelles compétences ou de prendre en considération les nouvelles interprétations que permettent les méthodes informatiques. Ils affirment aussi que les chercheurs récalcitrants utilisent parfois les humanités numériques comme prétexte pour régler d'anciennes rancunes personnelles ou de vieilles disputes entre disciplines. En outre, s'il est peut-être vrai que le monde universitaire aux États-Unis s'est ouvert aux fonds privés au cours des dernières années, on ne saurait imputer à l'émergence des humanités numériques ni les coupes budgétaires, aussi bien au niveau fédéral qu'au niveau des états, ni la réduction du soutien des fondations à l'éducation. Même si les chercheurs en sciences humaines et les programmeurs n'avaient pas commencé à se rapprocher, l'aggravation de la dette des étudiants et les promesses trompeuses des universités à but lucratif seraient tout aussi réelles. Et les résultats des projets en humanités numériques dans des domaines tels que l'analyse automatisée des textes, la visualisation de données, la cartographie géo-spatiale et l'analyse des réseaux n'ont fait que nourrir les échanges déjà riches au sein des disciplines des sciences humaines comme entre celles-ci. 
Ce débat se poursuivra sans aucun doute encore un certain temps, compte tenu des contraintes financières qui pèsent sur l'enseignement supérieur, aux États-Unis et ailleurs, et du rôle politique, économique et culturel assigné au secteur éducatif du $\mathrm{XXI}^{\mathrm{e}}$ siècle. La polémique se concentre davantage sur les espoirs pour le présent - et pour le futur - de l'université que sur une évaluation rigoureuse des mérites réels des recherches qui s’appuient sur des méthodes numériques. Dans cet essai, je voudrais présenter une manière d'évaluer le Projet $\underline{\mathrm{RCF}}$, et implicitement d'autres travaux en humanités numériques. Mon enquête s'appuie davantage sur une comparaison chronologique que sur les débats agités du climat politique actuel. Plus précisément, je souhaiterais examiner trois entreprises antérieures de quantification des registres de recettes tenus par la troupe de la Comédie-Française, qui sont au cœur du Projet $\underline{\mathrm{RCF}}$. Chacune de ces entreprises a été modelée par la conjoncture historique dans laquelle elle s'inscrivait, tout comme le Projet $\underline{\mathrm{RCF}}$ et plus généralement les projets en humanités numériques sont aujourd'hui soumis aux intérêts et aux besoins du temps présent. La première tentative est celle de Charles de Fieux, Chevalier de Mouhy (1701-1784), qui publia les Tablettes dramatiques et une série de suppléments de 1752 à 1758 en se fondant entre autre sur les registres de la troupe. Son but était de proposer une histoire du théâtre que les spectateurs du XVIII ${ }^{\mathrm{e}}$ siècle pourraient utiliser. Plus d'un siècle plus tard, Alexandre Joannidès (1879-1927), chercheur assidu qui passa plus de vingt-cinq ans à étudier les comptes quotidiens de la ComédieFrançaise, publia en 1901 les données contenues dans les registres sous une forme tabulaire. Par la suite, pendant deux décennies, il proposa annuellement des suppléments sur les activités de la troupe. En 1920, il donna une version revue et corrigée de son premier ouvrage et poursuivit ses mises à jour jusqu'à sa mort en 1927. Ces travaux permirent à la troupe de concevoir et d'exposer clairement le rôle commercial et culturel qui continuait à être le sien dans le paysage théâtral français du début du XX $\mathrm{XX}^{\mathrm{e}}$ siècle. Enfin, Henry Carrington Lancaster (1882-1954), professeur de langues romanes à l’Université Johns Hopkins, publia en 1941 puis en 1951 des transcriptions partielles des registres, couvrant la période de 1680 à 1774. Cette entreprise s'inscrivait dans un projet plus vaste d'histoire de la « Littérature dramatique française » des XVII ${ }^{\mathrm{e}}$ et XVIII ${ }^{\mathrm{e}}$ siècles. Lancaster était un membre important du corps professoral américain, et son intérêt pour les registres de la Comédie-Française et la scène théâtrale française d'Ancien Régime est étroitement lié aux efforts que firent d'autres académiciens de premier plan pour ancrer la démocratie et la culture américaines dans un héritage européen qu'ils estimaient source de valeurs universelles. En me penchant sur cette longue histoire des transcriptions et des interprétations des registres, je souhaite parvenir à une approche 
critique plus fine du Projet $\underline{\mathrm{RCF}}$ et, plus largement, des travaux en humanités numériques en cette seconde décennie du $\mathrm{XXI}^{\mathrm{e}}$ siècle.

\section{Charles de Fieux, Chevalier de Mouhy : un plumitif au service de l'histoire du théâtre}

Lorsque le chevalier de Mouhy publia ses Tablettes dramatiques en 1752, la ComédieFrançaise existait déjà depuis près de trois quarts de siècle $\underline{3}$. À la suite de la création de la troupe en 1680, Louis XIV lui avait octroyé le monopole des représentations payantes à Paris pour toutes les pièces en langue française. Aucune autre troupe n'avait l'autorisation de mettre en scène dans la capitale les œuvres de Molière, de Racine, de Corneille ou de tout autre dramaturge écrivant en français. Les ComédiensFrançais recevaient une pension royale annuelle, en compensation de laquelle ils devaient se rendre à la cour pour jouer selon le bon vouloir du roi. Cette combinaison efficace d'un monopole commercial public et du patronage royal attira aux spectacles donnés par la troupe à Paris aussi bien des membres de la cour et des dignitaires étrangers en visite que des auteurs et des intellectuels. Les échos des sujets controversés qui étaient représentés sur scène pour des publics socialement hétérogènes venant au spectacle chaque soir résonnaient bien au-delà de l'enceinte du théâtre. Dès le milieu du XVIII ${ }^{\mathrm{e}}$ siècle, la Comédie-Française était ainsi devenue une institution culturelle et politique majeure de la France des Lumières.

À cette époque déjà, un certain nombre d'auteurs et d'éditeurs avaient proposé des histoires empiriques du théâtre français et, plus généralement, des spectacles à Paris. Dès les années 1730, deux histoires en plusieurs volumes de la scène française parurent, visant à expliquer de manière plus rigoureusement chronologique l'ascension du théâtre français . La première, intitulée Recherches sur les théâtres de France, depuis l'année onze cens soixante-un, jusques à présent, fut réalisée par PierreFrançois Godard de Beauchamps, dramaturge de la Comédie-Italienne devenu historien. Elle découpait l'histoire de la scène française en fonction des vies de ses représentants les plus fameux $\underline{5}$. Lannée précédente, les frères Claude et François Parfaict publièrent le premier des quinze volumes de petit format qui constitueraient leur Histoire du théâtre français depuis son origine jusqu'à présent. À la différence des Recherches de Godard toutefois, la grande ampleur de ces quinze volumes permit aux deux frères d'offrir bien plus de détails historiques et critiques sur chaque pièce et sur chaque auteur pris en compte $\underline{6}$. La préface du dernier volume (1749) en promettait 
trois autres à paraître afin d'arriver jusqu'au temps présent. Ces derniers volumes de l'Histoire ne se concrétisèrent cependant jamais, ce qui laissa le champ libre à d'autres auteurs souhaitant écrire l'histoire contemporaine de la Comédie-Française.

Entre 1752 et 1758, Charles de Fieux, Chevalier de Mouhy, profita de cette opportunité pour réaliser les Tablettes dramatiques suivant un format nouveau qui devait, selon le pari de l'auteur et de ses éditeurs, plaire aux amateurs de théâtre à la recherche d'une synthèse facile d'accès sur le passé théâtral du pays. Mouhy était né dans une petite famille militaire à Metz en 1701 et il était le neveu d’Hilaire-Bernard de Longepierre, un dramaturge dont la tragédie Médée, ou Médée et Jason (1694) demeura au répertoire de la Comédie-Française durant tout le XVIII ${ }^{\mathrm{e}}$ siècle $\underline{7}$ [Fig. 1]. Mouhy et sa femme, elle aussi d'un lignage modeste, avaient établi leur résidence à Paris dès le milieu des années 1730 avec leurs cinq enfants. Par la suite et jusqu'à sa mort en 1784, le Chevalier subvint à ses besoins grâce à ses talents littéraires ainsi qu'à ses relations sociales et professionnelles. Il publia La Paysanne parvenue, Les Mille et une faveurs, Le Masque de fer et des douzaines d'autres romans, dont certains sont toujours étudiés aujourd'huiö. Durant les décennies 1730 et 1740, il entretint une correspondance régulière avec Voltaire, à qui il servit même de prête-nom pour l'un des pamphlets polémiques que le philosophe publia à la fin des années 1740 . Il fit paraître des gazettes manuscrites relatant les nouvelles et les rumeurs qu'il avait recueillies dans les salons et les cafés de la capitale, allant jusqu'à mettre son réseau au service de la police de Paris et jusqu'à lui servir d'informateur durant les années 1740. En dépit de ces relations, il fut enfermé à la Bastille par deux fois : la première en 1741 pour avoir publié un roman sans la permission du gouvernement et la seconde en 1745 parce qu'il avait fait circuler des bulletins de nouvelles sans autorisation officielle. Il travailla en collaboration étroite avec des éditeurs pour réaliser et vendre des récits s'inspirant de l'actualité et des ouvrages non fictionnels conçus pour être des succès commerciaux. Les informations que l'on trouve dans les préfaces et les pages de titre indiquent qu'il a parfois vendu ces textes imprimés directement chez lui. Pour résumer, Mouhy parvint à maintenir une stabilité financière pendant plus d'un demi-siècle en mettant à profit, par tous les moyens possibles, sa formation militaire, sa production littéraire médiocre mais prolifique, ses relations avec les éditeurs parisiens, ainsi que ses réseaux sociaux et culturels. Il fut ce que des historiens culturels de la France du XVIII ${ }^{\mathrm{e}}$ siècle ont appelé des «Grub Street writer », ou plumitifs - mais ce terme ne rend pas justice à la complexité des activités et des réseaux de Mouhy $\underline{9}$. 
Au début de la décennie 1750, ayant remarqué l'enthousiasme grandissant pour l'histoire du théâtre qu'avait généré des écrivains tels que Godard et les frères Parfaict et en l'absence de travaux récents sur le sujet, Mouhy se lance dans une nouvelle entreprise. Il tire parti de ses liens avec Voltaire et d'autres personnalités dans le monde du théâtre, et peut-être aussi de la présence continue de la Médée de Longepierre, son oncle, au répertoire de la Comédie-Française, pour commencer l'ébauche de ce qui s'intitulerait plus tard Tablettes dramatiques $\underline{10}$. Dans la préface de son livre publié en 1752, Mouhy explique qu'il s'est fixé pour but de fournir un « dictionnaire » des pièces de théâtre pouvant servir d'œuvres de référence aux côtés des histoires chronologiques de Godard et des Parfaict. Pour parvenir à un dictionnaire des pièces en langue française le plus complet et précis possible, il s'est tourné vers différentes sources d'information : les dramaturges les plus fameux de son temps, les comédiens de la Comédie-Française et les élites sociales (les gens du monde). Une fois dressée la liste de ces sources multiples, il s'empresse d'ajouter qu'il n'est pas à l'origine de nombre des observations que l'on trouve dans la suite de l'ouvrage, bien qu'elles demeurent non attribuées par respect pour ses interlocuteurs.

Aucune de ces approches n'était particulièrement surprenante ou atypique. Cependant, au milieu de ces précisions et de ces précautions, Mouhy indique qu'il a consulté les registres quotidiens de la troupe de la Comédie-Française pour déterminer combien de fois chaque pièce présente au répertoire avait été donnée avant de tomber. Mouhy avait conscience que la mesure qu'il avait choisie pouvait être discutée. Il rappelle que les frères Parfaict furent critiqués pour avoir précisé dans leur histoire du théâtre le nombre de représentations d'une pièce à sa création. Il observe que le public et les critiques autorisés, les « connoisseurs », ne jugent pas toujours des mérites d'une œuvre à l'aune de son succès initial et que les « cabales » ont souvent conspiré pour en faire tomber une. En outre, écrit-il, les amateurs de théâtre pourraient aisément nommer des pièces qui triomphèrent lors de leur création sans être jamais reprises ou, à l'inverse, d'autres qui tombèrent rapidement mais qui rencontrèrent par la suite un succès critique et commercial quand elles furent reprises. Bref, l'approche quantitative n'est pas une mesure parfaitement sûre de la qualité dramatique d'une œuvre, mais le mal que se donne Mouhy pour répondre aux objections qu'elle pouvait susciter en raison de ses lacunes supposées suggère qu'il leur accordait néanmoins du crédit.

Cette impression est confirmée quand on compulse la partie principale de ce travail de 400 pages : le « Dictionnaire » des pièces. Dans la préface, Mouhy insiste sur le format typographique inhabituel pour lequel il a opté. Il était difficile de résumer toute 
l'histoire de la scène française en un seul volume transportable et abordable financièrement, comme le savaient bien les lecteurs des imposants travaux de Godard et des frères Parfaict. Après plusieurs essais typographiques, Mouhy et ses éditeurs étaient toutefois arrivés à une solution ressemblant à un tableau, qui devait permettre aux lecteurs de saisir du premier coup d'œil les éléments essentiels sur une pièce donnée. Pour les œuvres dramatiques méritant une présentation plus ample, quelques phrases ou un court paragraphe suivent la première ligne présentée de manière tabulaire.

L'étude de deux pages du « dictionnaire » des pièces qui constitue la partie centrale des Tablettes de Mouhy permet de comprendre comment fonctionne en pratique ce dispositif typographique. Dans le premier exemple, au début de la liste alphabétique des pièces, des lignes noires donnent un cadre à la page et soulignent l'organisation des données sous forme de tableau [Fig. 2]. Sous le titre qui occupe la moitié haute, l'éditeur a précisé le champ alphabétique (ABE-ABS) et donc l'ordre des informations sur la page. Au-dessous, il a indiqué les cinq catégories de données fournies pour chaque entrée : le titre, l'auteur, la date de la première, le nombre de représentations à la création et la date de première publication. Ces cinq catégories, ainsi que les lignes qui séparent chaque catégorie et les organisent en colonne, sont ensuite renseignées pour les cinq pièces répertoriées ici. De manière générale, ces choix de mise en page encouragent le lecteur à parcourir pour chaque pièce les colonnes contenant des informations sur les représentations et les éditions. Seuls de brefs résumés d'une ligne ou deux apportant quelques précisions viennent arrêter le regard dans son mouvement vertical, ce qui permet de ne pas donner trop d'importance aux descriptions narratives, qui constituaient inversement un élément central des travaux de Godard et des frères Parfaict. 
Le second exemple, où sont répertoriées des pièces appartenant au champ alphabétique MAR, renforce l'effet tabulaire tout en permettant de voir l’hétérogénéité des données présentées [Fig. 3]. Cette page contient des informations sur neuf pièces. Seule la première colonne, qui donne le titre des pièces (Noms des Piéces.), est systématiquement renseignée. Un lecteur qui passerait en revue la seconde colonne, qui fournit le nom des dramaturges (Noms des Auteurs.), remarquerait rapidement que les auteurs de quatre pièces sont inconnus.

La troisième colonne indiquant l'année où a eu lieu la première représentation (An. Des Repr.) nous renseigne sur le fait que deux des neuf pièces n'ont jamais été données en public. Un coup d'œil à la colonne suivante sur la droite, proposant le nombre des

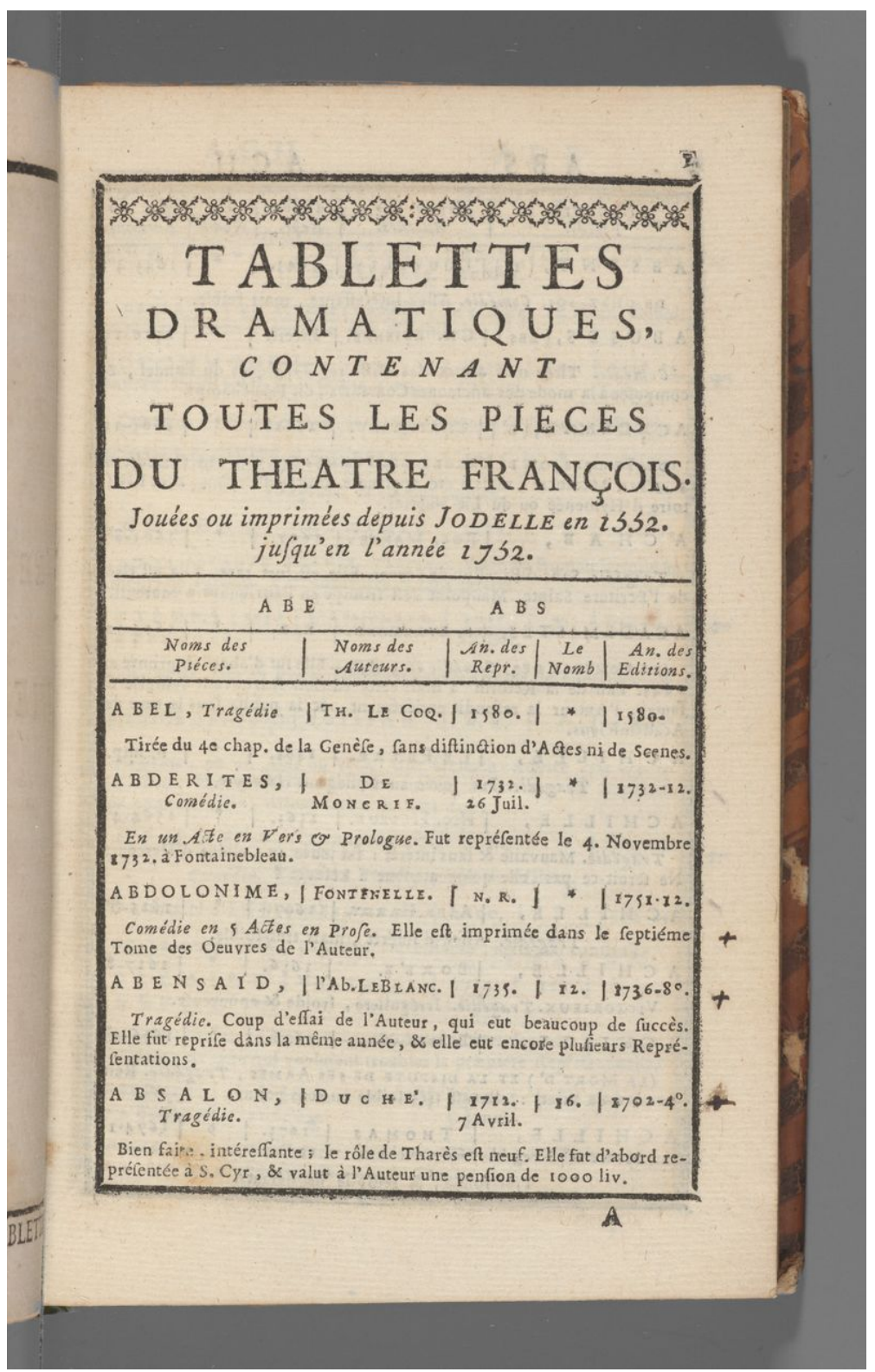

Figure 2. Charles de Fieux, Chevalier de Mouhy, Tablettes dramatiques, Paris, Jorry, 1752, p. 1. TS 375 13.5, Bibliothèque Houghton, Université Harvard.

représentations lors de la

création ( $L e N o m b$ ), permet de voir qu'aucune information concernant le succès des créations n'est disponible pour cinq des pièces. Dans chaque cas, la première a eu lieu avant que les troupes parisiennes ne commencent à enregistrer des informations sur les représentations. Enfin, la dernière colonne, qui propose la date et le format d'édition pour chaque pièce (An. Des Editions), révèle que quatre des neuf pièces n'ont 
pas été publiées ou bien que l'existence d'une version imprimée était incertaine. Aujourd'hui, on dirait que la série de données de Mouhy concernant ces neuf pièces est incomplète.

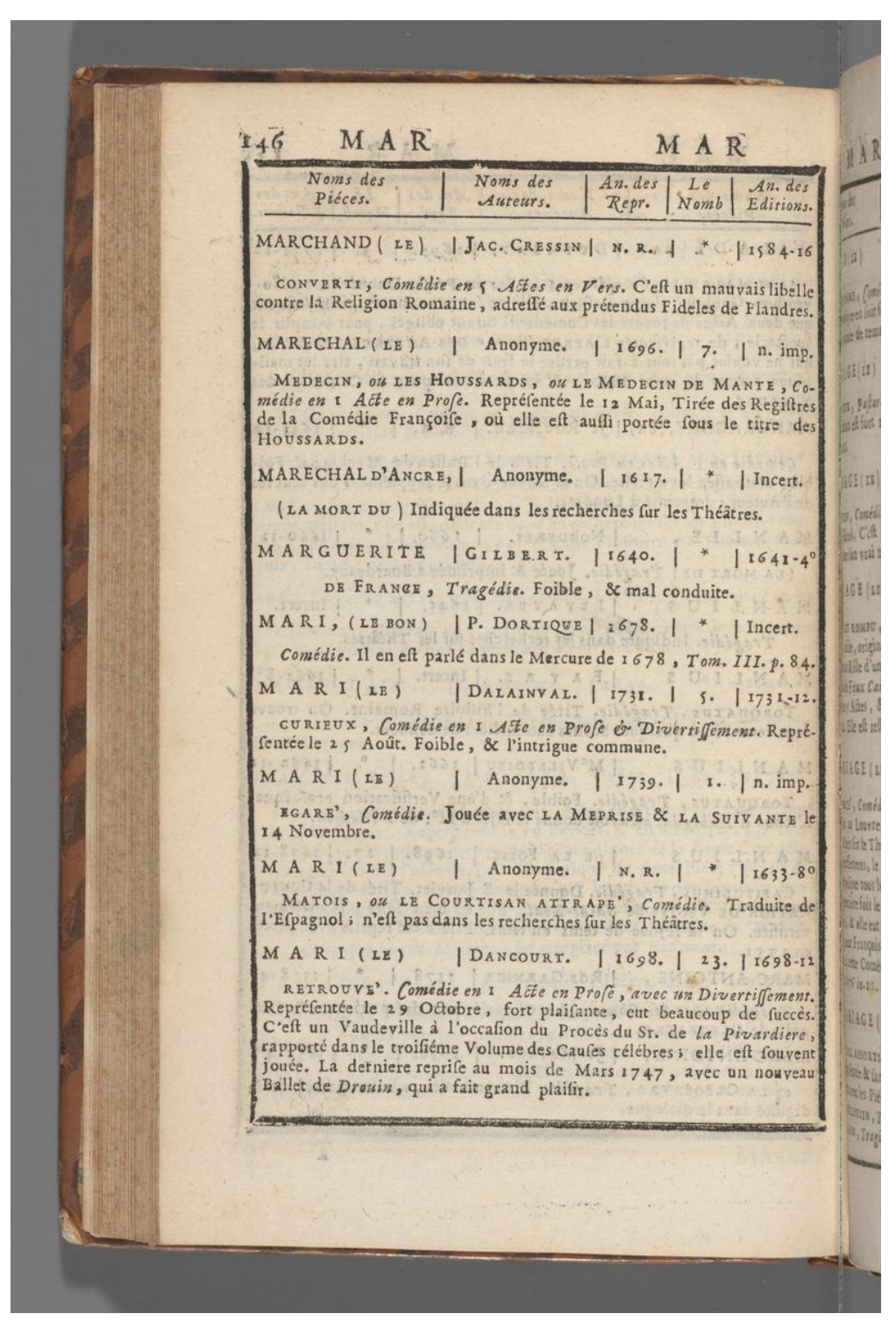

Figure 3. Charles de Fieux, Chevalier de Mouhy, Tablettes dramatiques, Paris, Jorry, 1752, p. 146. TS 375 13.5, Bibliothèque Houghton, Université Harvard.
Un lecteur moins pressé, ou qui serait à la recherche d'autres détails, pourrait poursuivre au-delà du parcours vertical de cette page et s'intéresser aux résumés proposés immédiatement au-dessous de l'entrée tabulaire qui donne, sur une seule ligne, des informations pour chaque pièce. Ces résumés vont du commentaire bref et dépréciatif (la tragédie de Gilbert représentée en 1640 Marguerite de France est « faible, \& mal conduite ») à un examen étendu de la pièce la plus connue de la page, $L e$ Mari retrouvé de Dancourt, qui connut 23 représentations lors de sa création en 1698. Dans ce dernier exemple, Mouhy nous apprend que la pièce était inspirée d'un procès bien connu à l'époque et que sa reprise la plus récente, en mars 1747, avait été agrémentée d'un nouveau ballet qui avait ravi les spectateurs 11 . Bref, un lecteur se reportant à cette page pour y chercher des informations sur une pièce appartenant au champ alphabétique MAR apprendrait rapidement ce qu'il y avait à savoir sur 
l'auteur de la pièce, ses premières représentations et son histoire éditoriale. En outre, il pourrait découvrir d'autres détails sur la réception de la pièce ou ses qualités esthétiques, tels que son genre, le nombre d'actes et son style (vers ou prose). Mouhy et ses éditeurs ont conçu un ouvrage de référence reposant sur une présentation tabulaire afin de fournir un instantané de l'histoire de chaque pièce, dans leurs versions scéniques et imprimées. Toutefois, ils choisirent de ne pas se reposer uniquement sur ce mode de présentation tabulaire parce que les lecteurs étaient aussi habitués aux résumés narratifs mettant l'accent sur les qualités formelles des textes théâtraux. Ce format hybride suggère une tension entre les stratégies interprétatives quantitatives et qualitatives qu'utilisaient les spectateurs de théâtre du milieu du XVIII ${ }^{\mathrm{e}}$ siècle.

Bien qu'il soit difficile de savoir comment les lecteurs traitaient en pratique les informations disposées sur les pages des Tablettes dramatiques, Mouhy lui-même indique dans une publication ultérieure avoir envisagé que son travail serait surtout utile comme référence pour les spectateurs qui, avant de se rendre au théâtre, voudraient se renseigner sur les pièces jouées :

[...] le premier besoin des personnes qui vont habituellement au Spectacle est, avant de s'y rendre, d'être instruites de ce qui a rapport aux Pieces qui doivent y être représentées : en jettant les yeux sur [ce livre], ils apprendront, au coupd’oeil, le temps de leurs representations, combien elles en ont eues, les noms de leurs auteurs $[\ldots] \underline{12}$

En d'autres termes, il visait à composer une histoire du théâtre pratique et facile à consulter pour des spectateurs qui souhaiteraient situer dans le contexte plus large du passé théâtral français une soirée à la Comédie-Française. L'analyse des Tablettes que fait le critique littéraire Élie-Catherine Fréron en 1752 confirme que les contemporains estimaient que le format typographique de l'ouvrage accomplissait cette mission. Fréron remarque que Mouhy a réussi à rassembler en un seul volume tout ce que « l'histoire de notre Théâtre peut offrir d'intéressant ». Le critique explique ensuite en détail la disposition des données concernant les pièces sur une ligne, si caractéristique du dictionnaire des œuvres conçu par Mouhy, et il conclut en faisant les louanges de l'auteur et de ses éditeurs, qui sont parvenus à condenser les informations importantes en un volume unique $\underline{13}$.

L'enthousiasme de Fréron pour la forme des Tablettes de Mouhy semble avoir été partagé par les lecteurs, si l'on en juge à l'aune du succès commercial de l'ourvrage. 
La première édition de 1752 coûtait six livres. À titre de comparaison, l'édition imprimée au format in-12 d'une pièce seule ou un billet autorisant à se tenir debout dans le parterre de la Comédie-Française coûtaient en général une seule livre durant la majeure partie du XVIII ${ }^{\mathrm{e}}$ siècle. L'achat de l'édition de 1752 donnait le droit de recevoir annuellement une révision imprimée gratuite que Mouhy et ses éditeurs faisaient paraître chaque année avant Pâques, au moment des trois semaines de relâche séparant les saisons théâtrales. Les lecteurs pouvaient aller chercher ces suppléments chez Mouhy ou chez ses éditeurs. Pour ceux qui n'avaient pas acheté le recueil original de 1752, les addenda annuels revenaient à douze sous, soit trois cinquièmes d'une livre. Lorsque arriva la relâche de la Pâque 1755, Mouhy dut presser les lecteurs de venir chercher leurs suppléments dès leur parution parce que les éditeurs se trouvaient rapidement à court d'exemplaires. Les années suivantes, ils promirent d'en publier deux fois plus.

Mouhy et ses éditeurs Pissot, Jorry, Duchesne et Lambert (ce dernier se trouvant dans la même rue que le théâtre de la Comédie-Française) firent paraître ces suppléments durant six ans, jusqu'en 1758. Cette année-là, Louis XV nomma à la tête du ministère de la guerre le Maréchal Duc de Belle-Isle, qui était déjà le protecteur de Mouhy. BelleIsle avait besoin d'yeux et d'oreilles pour se tenir informé des nouvelles de la capitale et il offrit à Mouhy une pension en échange de la promesse qu'il arrêterait de publier ses travaux sur l'histoire du théâtre ou sur tout autre sujet. De plus, il est vraisemblable que le supplément, qui se limitait à rapporter les nouvelles concernant la saison la plus récente de la Comédie-Française, ne parvenait plus à rivaliser avec un almanach du théâtre qui commença lui aussi à paraître au début des années 1750 chaque année $\frac{14}{}$. À la fin de la décennie, celui-ci proposait des nouvelles fraîches et des anecdotes sur tous les spectacles de la capitale dans un format plus petit et plus facilement transportable, et sans qu'il ne soit besoin de lui adjoindre en complément l'édition de l'année précédente. Toutefois, les almanachs ne proposaient pas une synthèse des informations se trouvant dans les registres de la Comédie-Française et ils ne se présentaient pas sous le format typographique qui caractérisait les Tablettes et leurs suppléments.

Mouhy et ses éditeurs semblent avoir essayé de couvrir leurs arrières grâce à ce dispositif tabulaire avant même que la parution annuelle des almanachs ne devienne un événement éditorial attendu à la fin des années 1750. En 1752, la ComédieFrançaise imprima une liste des pièces de son répertoire à usage interne, le Calendrier pour l'année MDCCLII à l'usage des comédiens François ordinaires du roi $\underline{15}$. Ce document n'était pas destiné à être vendu au public mais quand Mouhy en obtint une 
copie, il commença à s'inquiéter du fait que ses Tablettes ne contenaient pas une liste précise de toutes les pièces du répertoire. C'est pourquoi il publia avec ses trois éditeurs un nouvel ouvrage en 1753, Le Répertoire de toutes les pièces restées au Théâtre François, avec la date, le nombre des représentations, et les noms des Auteurs et des Acteurs vivans, dans un format plus petit et moins cher que les Tablettes $\underline{16}$. Ce nouvel ouvrage se passait du format tabulaire qui augmentait les coûts de production, incorporant au résumé de la pièce les informations sur le nombre de représentations et sur l'édition. Ainsi, Mouhy et ses éditeurs, dès le début des années 1750, visaient différentes niches du marché des histoires imprimées du théâtre. Quand le Chevalier se remit à l'écriture d'histoires du théâtre vers la fin de sa vie en 1780, le recueil étendu qu'il publia en trois volumes et dans un grand format, l'Abrégé de l'histoire du théâtre français, ne se présentait plus sous forme de tableaux. Il contenait bien des informations que l'on trouvait dans les premières éditions mais celles-ci étaient dorénavant présentées dans le corps d'un texte ordinaire, à l'exception du nombre de représentations à la création. On peut en déduire qu'en 1780 les lecteurs à la recherche d'informations sur le passé théâtral français n'avaient plus besoin de toutes les données ni du format tabulaire conçu par Mouhy et ses éditeurs.

\section{Alexandre Joannidès : « historiographe » de la Comédie- Française, 1901-1926}

Un siècle et demi plus tard, le paysage théâtral parisien dans lequel s'inscrit la troupe du Français avait évolué et était devenu plus compétitif. Le monopole commercial sur les pièces en langue française dont avait bénéficié la Comédie-Française depuis sa création avait disparu pendant la période révolutionnaire, entre les années 1790 et le début du XIX ${ }^{\mathrm{e}}$ siècle, mais Napoléon Bonaparte le remit en vigueur par une série de décrets en 1807 et 1808 . Les régimes suivants, qu'ils soient monarchiques ou républicains, maintinrent ces restrictions jusqu'à ce que Napoléon III, en 1864, les suspendent définitivement dans un mouvement général de libéralisation des pratiques commerciales françaises. La troupe dut ainsi par la suite rivaliser avec d'autres compagnies théâtrales qui avaient désormais le droit de jouer leur répertoire. LÉtat, toutefois, censurait toujours textes et représentations, et à la fin des années 1840 le gouvernement avait également insisté, en échange de son soutien matériel, pour nommer un administrateur général des affaires artistiques et budgétaires de la compagnie. À la fin du XIX ${ }^{\mathrm{e}}$ siècle, la troupe était en compétition au niveau financier avec d'autres compagnies de théâtre qui avaient les ressources nécessaires pour 
recruter des comédiens de talent et acheter les pièces des nouveaux auteurs à la mode. L'émergence du théâtre d'avant-garde dans les années 1880, souvent associée au Théâtre Libre d'André Antoine, engendra de nouveaux défis : pour éviter la censure d'état, ce dernier proposait une entrée gratuite quand il donnait des pièces au sujet polémique attirant spectateurs et critiques fin-de-siècle. De plus, le développement de nouvelles formes contribua à bouleverser le marché du divertissement : le caféconcert, le musée de cire, les dioramas, la presse imprimée, la photographie et, dans les années 1890, le cinéma, alors à ses balbutiements. Au début du XX ${ }^{\mathrm{e}}$ siècle, on ne voyait pas bien de quelle façon une institution dont les origines remontaient à l'époque du Roi-Soleil allait pouvoir s'adapter à cette nouvelle scène médiatique et commerciale $\underline{17}$.

En réponse à cela, la troupe du Français misa encore davantage sur son héritage historique. Depuis 1799, elle jouait en plein cœur de Paris, dans un théâtre de la rue de Richelieu à l'angle sud-ouest du Palais Royal, lieu qu'elle occupe toujours aujourd'hui. Ce bâtiment largement décoré ne se contentait pas de proposer des espaces de représentation et de répétitions ou des bureaux pour l'administration, mais il accueillait également les archives des mises en scènes rassemblées sur plusieurs générations ainsi qu'une collection en constant développement de sculptures, de peintures et d'autres objets dignes d'un musée. On y trouvait aussi une riche bibliothèque de textes de théâtre manuscrits ou imprimés. En 1879, le fauteuil dans lequel Molière s'était assis durant son dernier spectacle avant de mourir en 1673 ne fut plus utilisé sur scène et fut disposé dans le foyer du théâtre, à proximité de sculptures représentant les grands dramaturges et de portraits de comédiens appartenant à l'histoire de la troupe. La valeur commerciale de la Comédie-Française au tournant $\mathrm{du} \mathrm{XX}^{\mathrm{e}}$ siècle reposait surtout sur sa prétention à incarner un nationalisme culturel persistant, au côté d'institutions aussi bien établies que le Louvre ou l’Académie française. D’autres compagnies pouvaient bien offrir des spectacles exploitant la veine romantique ou nihiliste de la culture contemporaine, comme le Cyrano de Bergerac d'Edmond Rostand ou Ubu roi d'Alfred Jarry, mais aucune ne pouvait égaler le poids de la tradition historique qui se donnait à voir rue de Richelieu. En 1900, la Comédie-Française n'était pas uniquement la somme de son répertoire vieux de 220 ans mais elle offrait aussi une sorte de musée vivant du théâtre où les interprètes et les dramaturges présents étaient en dialogue permanent avec les artistes du passé. Ainsi que l'écrit un critique en 1902, c'était « une illustre maison qui, seule entre nos théâtres, avec ses toiles et ses bustes, ses livres et ses archives, son auréole de gloire solennelle et souriante, les noms pompeux et galants qui forment 
la chaîne de son histoire, a le privilège de satisfaire les goûts de l'érudit et ceux du dilettante $\underline{18}$. »

Cet environnement faisant la part belle à l'histoire attira le jeune Alexandre Joannidès qui avait émigré à Paris [Fig. 4]. Il était né en 1879 à Manchester, en Angleterre, d'un père grec et d'une mère anglaise. Joannidès avait sept ans quand son père mourut et que sa mère partit s'installer avec sa famille à Paris $\underline{19}$.



Figure 4. Albert Braïtou-Sala, Portrait d'Alexandre Joannidès à l'âge de 40 ans, 1919. Avec l'aimable autorisation de la BibliothèqueMusée de la Comédie-Française.
En 1892, il commença à étudier la peinture. Un de ses oncles, du même calibre que Mouhy, vivait lui aussi à Paris et était dramaturge. Au milieu des années 1890, les ambitions picturales du jeune Alexandre arrêtèrent l'attention d'Henri Cain, artiste réputé et auteur de livrets d'opéra, de pièces dramatiques et de poèmes. Sous son égide, Joannidès étudia la peinture et développa aussi une passion pour le théâtre. Il prit l'habitude d'assister aux matinées à la ComédieFrançaise et ailleurs, et il se mit à collectionner les affiches, les programmes et les photographies des comédiens les plus en vue du Français. Cain finit par présenter son jeune protégé à son ami Jules Clarétie, l'administrateur général de la Comédie-

Française. Grâce à ce dernier, Joannidès rencontra ensuite l'archiviste de la troupe, Georges Monval, qui lui donna accès à la bibliothèque et aux archives. Plongé dans les dossiers poussiéreux de la troupe, Joannidès conçut bientôt le projet de combler un manque dans l'histoire de la compagnie et de réaliser une synthèse systématique de la longue histoire des représentations du théâtre sur deux siècles. Pour ce faire, il décida 
de s'appuyer sur les informations contenues dans les registres annuels des recettes, remontant à 1680, soit la même source que Mouhy avait consultée un siècle et demi auparavant de manière plus désordonnée $\underline{20}$.

Fondé sur la mise en tableau méthodique des registres de la Comédie-Française, le premier ouvrage de Joannidès, le plus important aussi, intitulé La Comédie-Française de 1680 à 1900. Dictionnaire général des pièces et des auteurs, parut en $1901 \mathrm{chez}$ l'éditeur littéraire Plon-Nourrit, comme tous les volumes suivants sur le même sujet, sous la forme d'une édition limitée de deux cent cinquante exemplaires. Dans sa préface, Clarétie signale les circonstances difficiles dans lesquelles le jeune historien réalisa son travail. En mars 1900, un feu avait éventré le somptueux théâtre, rue de Richelieu. Bien que la plupart des collections de peintures et de sculptures de la compagnie ait été sauvée, avec ses archives volumineuses et sa bibliothèque, les comédiens durent trouver refuge ailleurs pendant les travaux de reconstruction. Les contemporains ne manquèrent pas de remarquer ce qu'il y avait d'ironique à voir ce théâtre consumé par les flammes au tournant du siècle, comme si la troupe n'avait désormais plus rien à apporter à l'identité nationale française. Cependant, Joannidès, qui avait commencé ses travaux plusieurs années avant l'incendie, acheva ses recherches dans les salles de consultation installées temporairement au Louvre, à quelques encablures du théâtre. D’une certaine manière, son travail vint consacrer ce passé, tout en promettant des mises à jour annuelles des tableaux et des statistiques concernant les pièces et les auteurs qui constituaient le précieux héritage de la troupe. Dès 1901, aussi bien la salle du théâtre que le répertoire légendaire furent repensés pour devenir les fondements de la pertinence de la compagnie dans le nouveau siècle. Ainsi que le remarque Clarétie lui-même dans sa préface à l'édition de 1901 :

Que de gloire accumulée de 1680 à 1900 ! [...] Elle est immortelle, elle est indestructible, la Maison de Molière, de Corneille, de Racine, de Victor Hugo, qui, sur sa façade offre désormais les profils de ces poètes à l'admiration des foules. Et c'est une pierre de plus ajoutée à sa gloire que le livre excellent et utile de M. Joannidès, historiographe de la Comédie-Française. $\underline{21}$

La Comédie-Française de 1680 à 1900 trouve ses racines dans le travail de Mouhy et des autres historiens du XVIII ${ }^{\mathrm{e}}$ siècle qui se sont intéressés au théâtre français. Jules Truffier, un des acteurs de la troupe, le rappelle dans une préface qu'il rédigea pour l'annuaire de Joannidès en 1903 : «J'ai vu un jour [...] un Agenda ou Almanach du Théâtre François, publié probablement en 1757, dans lequel un amateur avait, autrefois, esquissé le plan de votre entreprise $\underline{22} »$. Truffier confesse son ignorance au 
sujet du travail de cet auteur du XVIII ${ }^{\mathrm{e}}$ siècle, mais il note de façon approbatrice que « vous aussi, cher Monsieur Joannidès, vous doteriez un jour, au commencement du $\mathrm{XX}^{\mathrm{e}}$ siècle, les amis de l'Art dramatique de votre superbe agenda ». Les 450 pages de La Comédie-Française de 1680 à 1900 sont divisées en trois parties, que Mouhy et ses lecteurs n'auraient pas manqué de reconnaître. Elles comprennent une liste alphabétique longue de 88 pages recensant les titres de toutes les pièces représentées depuis 1680, avec les dates des premières, une autre avec les noms de tous les auteurs ayant écrit pour la troupe depuis 1680 ainsi que les titres de leurs pièces, et une énumération sur 246 pages des pièces jouées chaque année entre 1680 et 1900, avec le nombre de fois où elles furent représentées chaque saison. Ainsi, la partie principale du livre offrait le même type d'informations que celles que Mouhy et les éditeurs de l'almanach avaient compilées un siècle et demi plus tôt : un index des auteurs et des pièces du répertoire permettant de trouver aisément les informations concernant une œuvre ou un écrivain en particulier, ainsi qu'un découpage par année de ce répertoire. Bien sûr, la compilation de Joannidès avait le mérite d'inclure un siècle et demi de données supplémentaires.

En amont de ces trois listes détaillées qu'il se donna tant de mal à établir, Joannnidès ajouta un tableau qui dut apparaître comme novateur à ses lecteurs du début du $\mathrm{XX}^{\mathrm{e}}$ siècle et qui contribuait à asseoir la pérennité de la Comédie-Française en tant qu'institution. Alors que les expériences de Mouhy au milieu du XVIII ${ }^{\mathrm{e}}$ siècle mélangeaient textes et données, Joannidès ouvre son ouvrage avec onze tableaux fournissant des informations sur le nombre de fois où les pièces de quelques auteurs parmi les plus joués dans l’histoire de la troupe avaient été représentées par décennie, de 1680 aux années 1890. Celui sur Molière (1622-1673), par exemple, montre la distribution sur vingt-deux décennies des 20290 représentations de ses 32 pièces au répertoire. On y constate que même durant la seconde moitié du XIX ${ }^{\mathrm{e}}$ siècle, un grand nombre de ses œuvres continuaient à figurer au répertoire de la troupe [Fig. 5]. 


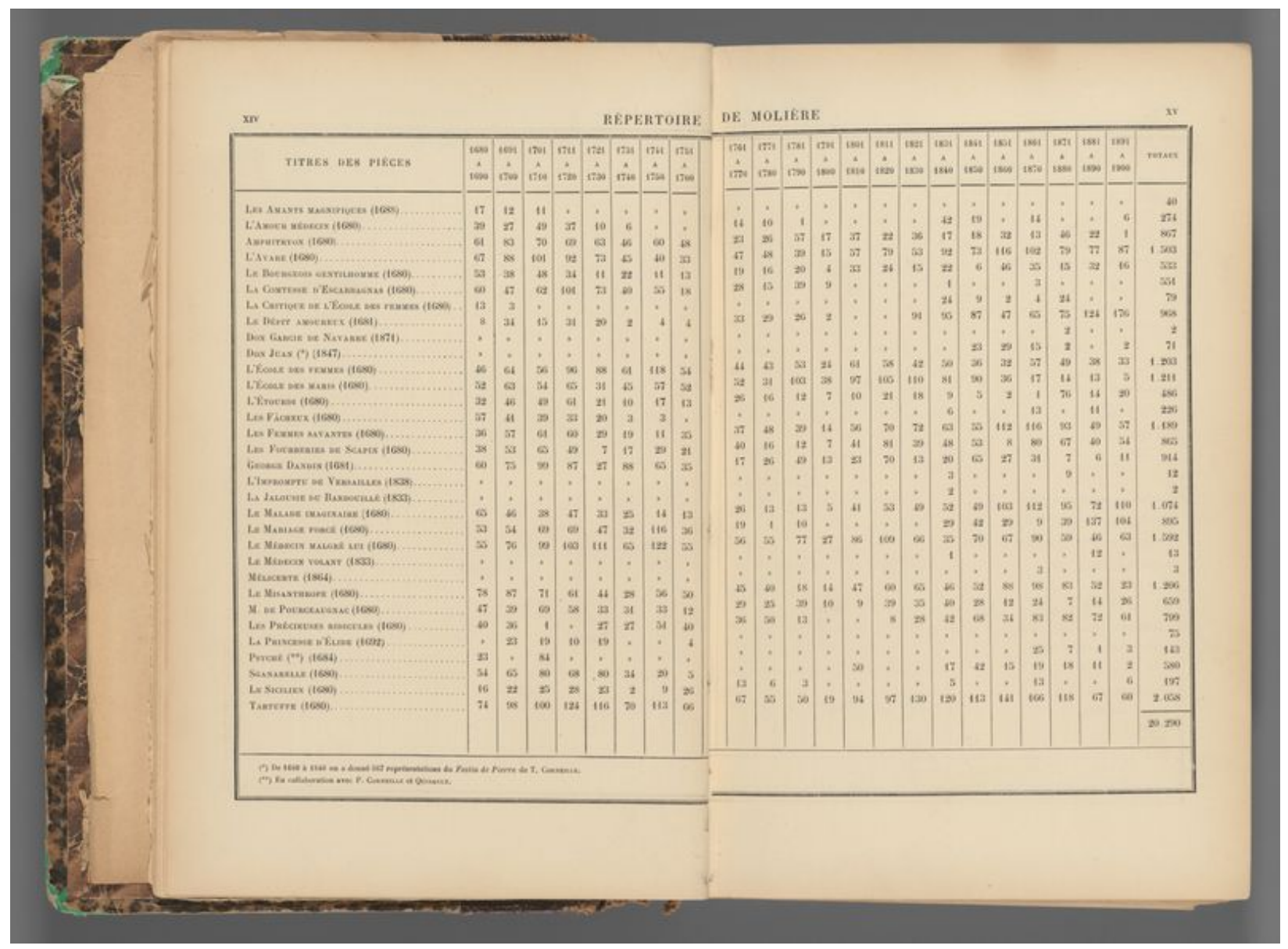

Figure 5. Alexandre Joannidès, La Comédie-Française de 1680 à 1900, Paris, Plon, 1901, p. xiv-xv.

Avec l'aimable autorisation de la Bibliothèque de l'Université Harvard.

On pourrait comparer ce tableau avec celui que Joannidès réalisa sur Voltaire (16941788), dont les 31 pièces furent représentées près de cinq fois moins que celles de Molière. La perte d'intérêt pour l'œuvre dramatique de Voltaire débute clairement avec les années révolutionnaires, durant la dernière décennie du siècle, puis s'amplifie de manière vertigineuse après $1850 \underline{23}$ [Fig. 6]. 


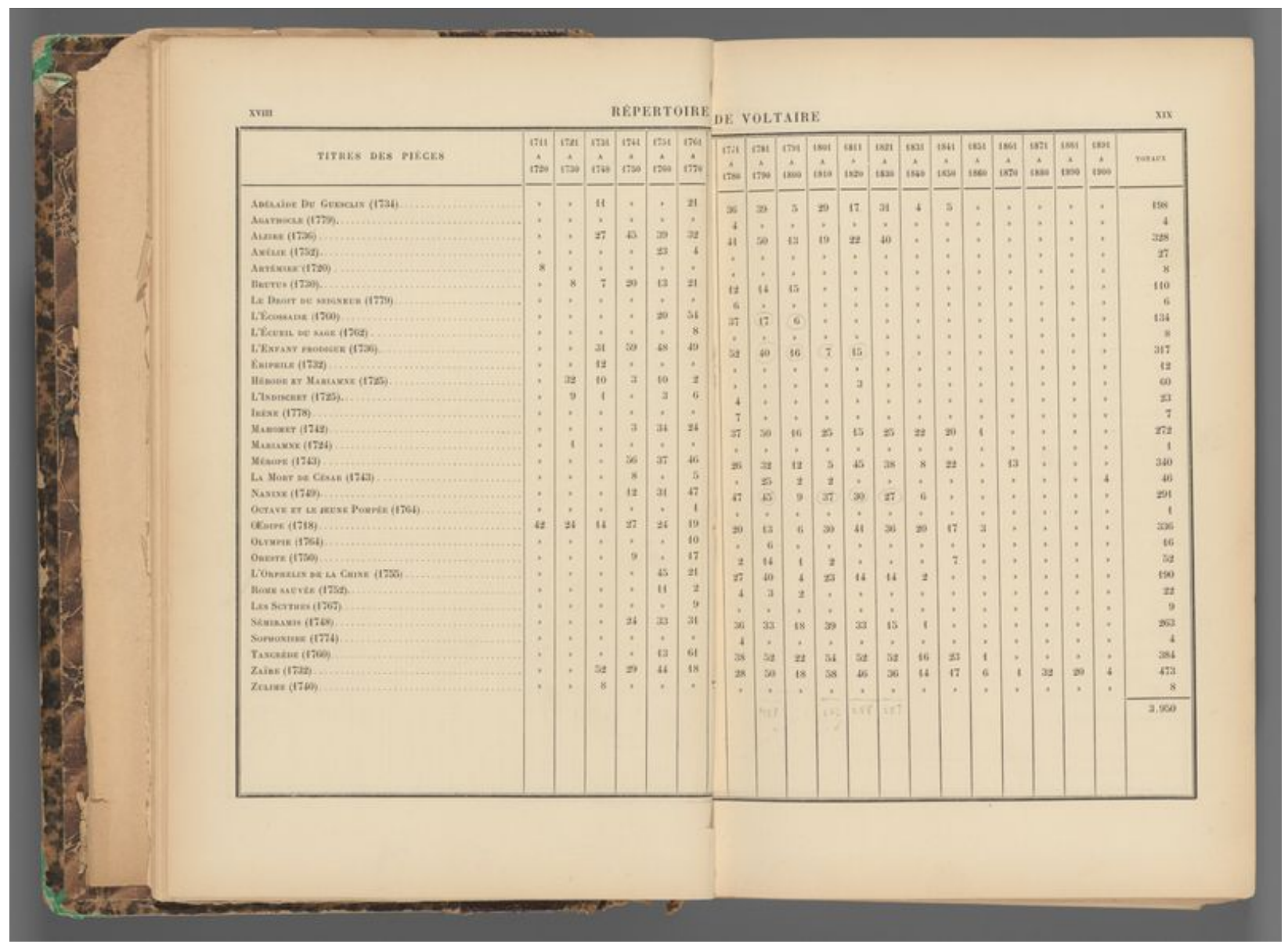

Figure 6. Alexandre Joannidès, La Comédie-Française de 1680 à 1900, Paris, Plon, 1901, p. xviii-xix.

Avec I'aimable autorisation de la Bibliothèque de l'Université Harvard.

Enfin, les quatre derniers tableaux que publie Joannidès synthétisent et donnent à voir la popularité de quatre auteurs du XIX ${ }^{\mathrm{e}}$ siècle : Victor Hugo (1802-1885), Alfred de Musset (1810-1857), Émile Augier (1820-1899) et Alexandre Dumas fils (1824-1895). Pour les deux premiers, les tableaux présentent des données concernant huit décennies et, pour leurs homologues légèrement plus récents, des statistiques sur six décennies [Fig. 7]. 


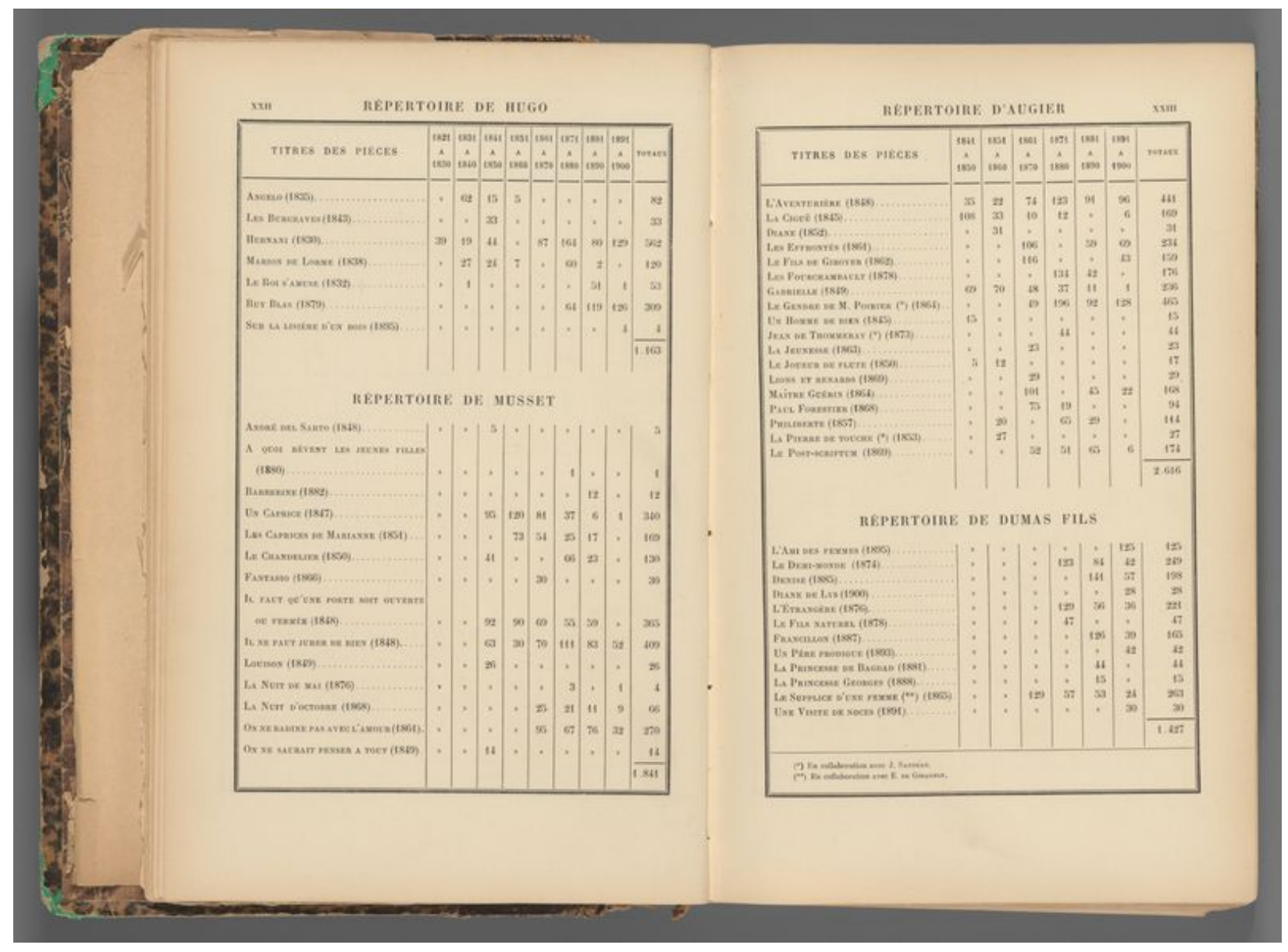

Figure 7. Alexandre Joannidès, La Comédie-Française de 1680 à 1900, Paris, Plon, 1901, p. xxii-xxiii.

Avec l'aimable autorisation de la Bibliothèque de l'Université Harvard.

Pour le XVII ${ }^{\mathrm{e}}$ siècle, Joannidès réalisa des tableaux similaires sur Pierre Corneille et sur Racine et, pour le XVIII ${ }^{\mathrm{e}}$ siècle, sur Regnard, Marivaux et Beaumarchais. Les six tableaux reproduits ici nous renseignent sur la manière dont les travaux de Joannidès contribuèrent à construire l'histoire de la Comédie-Française au tournant du $\mathrm{XX}^{\mathrm{e}}$ siècle. Si Molière constitue le socle de ce répertoire qui s'étend sur plus de deux siècles, l'ascension puis la chute du dramaturge le plus important pour la troupe au $\mathrm{XVIII}^{\mathrm{e}}$ siècle, Voltaire, apparaît clairement en contraste, et la popularité des quatre auteurs du XIX ${ }^{\mathrm{e}}$ siècle indique la «modernisation » du répertoire à cette période.

Ces tableaux montrent que le choix d'une description quantitative de la ComédieFrançaise est plus clair chez Joannidès qu'il ne l'était dans les Tablettes de Mouhy : comme le signale l'acteur Truffier dans la préface de 1902, Joannidès avait préparé 
une « œuvre mathématiquement historique $\underline{24}$ ». L'évaluation des pièces proposée par Mouhy après leur résumé sur une ligne a disparu, ce qui laisse aux lecteurs le soin de combiner et d'interpréter les lignes et les colonnes, sans l'aide d'une explication rédigée. La confiance accrue que Joannidès accorde aux synthèses statistiques est en partie le reflet de la durabilité de l'institution. Quand Mouhy publiait ses Tablettes et ses suppléments annuels dans les années 1750, la Comédie-Française n'existait que depuis trois générations, et sa place dans le paysage théâtral parisien était en constante évolution, en dépit du privilège royal dont bénéficiait la troupe. Les spectateurs réfléchissaient en termes de canon dramatique et cherchaient des informations sur les pièces et les auteurs afin de pouvoir débattre du positionnement de ces derniers dans le champ littéraire. La Comédie-Française jouait un rôle complémentaire dans l'établissement de cette hiérarchie. Au début du $\mathrm{XX}^{\mathrm{e}}$ siècle, la troupe pouvait s'appuyer sur une durabilité sans commune mesure. Bien sûr, beaucoup de grands auteurs du XIX ${ }^{\mathrm{e}}$ siècle avait cherché à accroître leur prestige en faisant jouer leurs textes par la prestigieuse institution mais c'était elle seule qui conférait ce prestige, ainsi que son musée qui faisait tous les soirs le récit de l'histoire du théâtre, rénové de manière somptueuse et se trouvant en face du Louvre, rue de Rivoli. Dans ce contexte, et à un moment plus favorable à l'analyse quantitative de la culture et des politiques publiques, les tableaux que Joannidès publiait au seuil de son grand œuvre de 450 pages se présentaient comme le résultat de recherches rigoureuses et offraient un nouvel éclairage sur cette légendaire institution culturelle $\underline{25}$.

Clarétie commente cette approche dans sa préface de 1901 à l'ouvrage de Joannidès, signalant que « [v]oilà de la statistique littéraire intéressante $\underline{26}$ ». Il observe que les tableaux de Joannidès montrent combien « en ce pays de France, le rire, la comédie est l'art représentatif du tempérament national ». La popularité au long cours de Molière, le plus grand auteur comique de la France, validait cette affirmation. Toutefois, les tableaux permettent également à Clarétie de remarquer que sur 220 ans, Racine est le second auteur le plus joué dans l'histoire de la troupe avec 6270 représentations, en partie grâce à son unique comédie, Les Plaideurs, qui à elle seule totalise un sixième de ces représentations et qui a été jouée plus souvent à la ComédieFrançaise que chacune des tragédies. Lauteur de comédies Jean-François Regnard, mort en 1709, a été représenté plus souvent au Français que Pierre Corneille et six de ses pièces se trouvent toujours au répertoire en 1890. De plus, bien que la pièce la plus populaire de Corneille durant plus de deux siècles fût la tragédie du Cid (1636), la seconde est sa comédie de 1644, Le Menteur. Bref, Clarétie s'appuie sur le travail de Joannidès pour mener un raisonnement tabulaire liant la longue histoire du théâtre au 
stéréotype en vogue de la gaieté française. Alors que la troupe avait développé une réputation de sérieux, son répertoire était également le reflet d'un amour du rire que les contemporains affirmaient être profondément ancré dans le caractère national.

Pendant les vingt années qui suivirent la première parution de ce travail monumental, Joannidès publia une mise à jour annuelle incluant des informations au sujet des nouvelles pièces, des nouveaux membres de la troupe, des événements remarquables et des évolutions administratives, ainsi que des critiques des spectacles de la saison précédente. Quand arrive 1920 cependant, suffisamment de données sur les nouvelles représentations avaient été accumulées depuis le premier ouvrage de 1901 pour que Joannidès décide de réviser sa somme statistique sur l'histoire de la troupe. Dans la préface de ce nouvel ouvrage, La Comédie-Française de 1680 à 1920. Tableau des représentations, Joannidès explique avoir décidé de suivre une nouvelle approche dans la présentation des données provenant des registres. Les tableaux qui se trouvaient au début de l'ouvrage de 1901 indiquaient le nombre total de représentations pour chacune des pièces écrites par onze auteurs qu'il avait choisis. Dans l'intervalle, les lecteurs avaient fait savoir qu'ils désiraient disposer des mêmes données pour toutes les pièces du répertoire, et pas seulement pour celles de Molière, de Racine et de quelques autres auteurs canoniques. Joannidès décida de répondre à ces critiques, tout en ajoutant des statistiques concernant les représentations des vingt années écoulées. Le nouvel ouvrage est débarrassé des onze tableaux qui figuraient en ouverture de l'édition de 1901 mais la nouvelle préface comprend deux listes mises à jour. L'une indique les trente auteurs dont les œuvres ont été représentées mille fois ou plus durant les 240 dernières années, classées par ordre décroissant. La seconde présente les 55 pièces ayant été jouées 500 fois ou plus depuis 1680, également par ordre décroissant [Fig. 8]. 


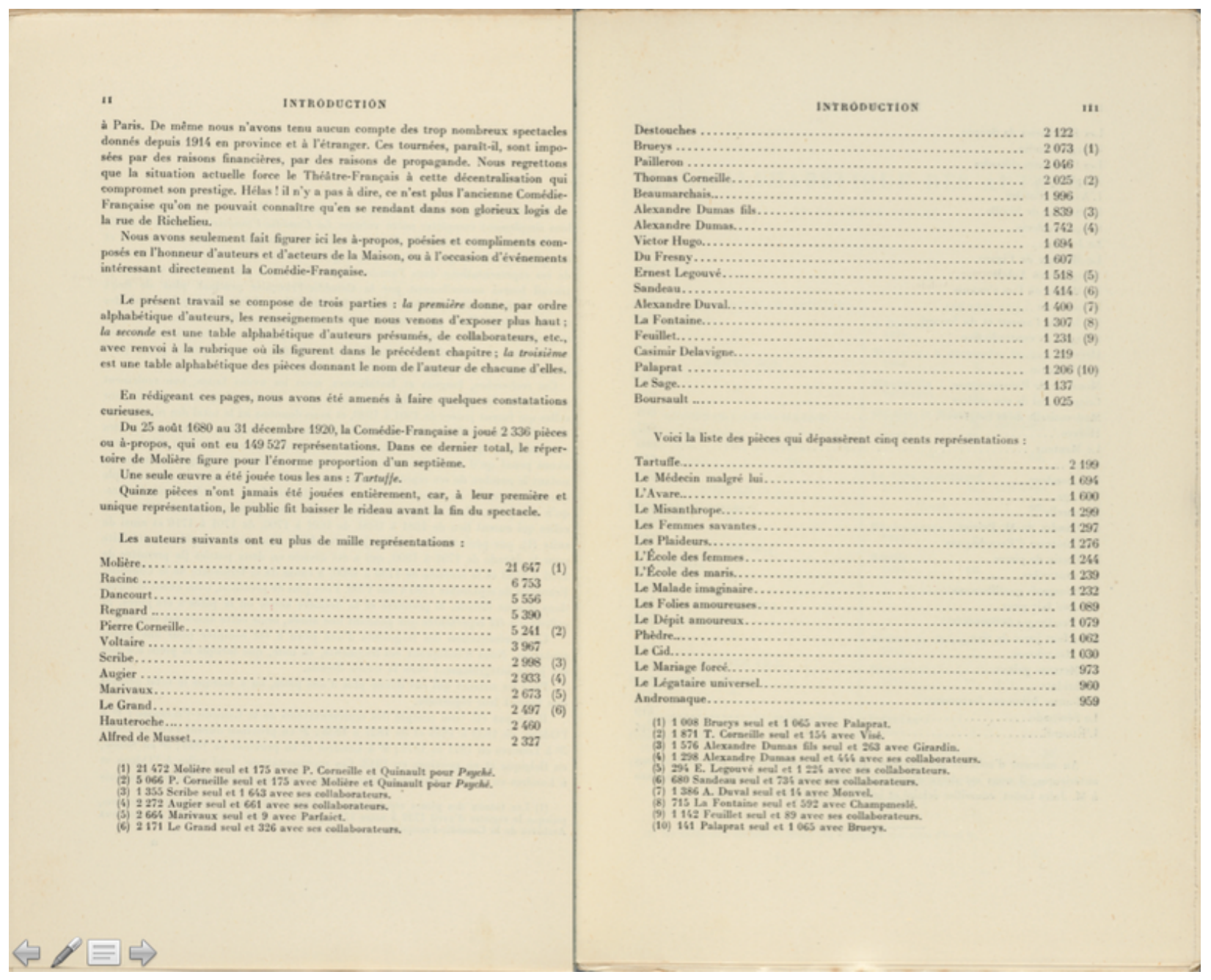

Figure 8. Alexandre Joannidès, La Comédie-Française de 1680 à 1920, Paris, Plon, 1921, p. ii-iii.

Harvard Theater Collection, Université Harvard.

Après la préface vient la partie la plus longue de l'ouvrage : la liste alphabétique des auteurs avec leurs pièces, mentionnées sous leur nom. En regardant par exemple l'une des pages dévolues au travail de Dancourt, on découvre la nouvelle mise en forme que propose la version de 1921 [Fig. 9]. On peut voir en bas de la première colonne une entrée pour la pièce en un acte Le Mari retrouvé (1698), inspirée d'une « cause célèbre » de l'époque également mentionnée par Mouhy dans ses Tablettes. Joannidès montre que la pièce se maintint dans le répertoire de la troupe jusqu'en 1835, pour un total de 373 représentations, soit plus que toutes les autres œuvres de l'auteur mentionnées sur cette page et plus que toutes les autres pièces de Dancourt au répertoire, à l'exception de deux. Joannidès réalise ce type de calcul pour les 2336 œuvres au répertoire de la troupe en 1920, ce qui occupe 106 pages des 137 que 
comporte l'ouvrage. Ces décomptes représentent une avancée conséquente par rapport au volume de 1901, qui indiquait le nombre de fois où une pièce avait été jouée sur une saison mais laissait aux lecteurs la tâche d'additionner les données sur plus de deux siècles afin de parvenir au nombre total de représentations pour chaque pièce. L'ouvrage de 1921 répond plus clairement aux besoins de ceux qui cherchaient une histoire quantitative plus complète des 240 années d'existence de la troupe.

Cette nouvelle « interface utilisateur », qui proposait un accès plus aisé à l'historique des représentations des plus de deux mille pièces mises en scène par la troupe depuis sa fondation en 1680, fit émerger de nouvelles réflexions chez ceux qui s’intéressaient au passé du théâtre français. Henri Bidou, un journaliste et homme de lettres qui écrivait dans le Journal des débats en 1921, remarque que le décompte des représentations décennie par décennie permet à Joannidès de présenter sous forme graphique l'histoire de chaque pièce par une « courbe devant les yeux $\underline{27}$ ». Bidou affirme que les tableaux correspondants sont non seulement une aide indispensable à tous ceux qui souhaiteraient écrire l'histoire du théâtre mais qu'ils proposent aussi « une lecture attachante et féconde en surprises ». Bidou porte une attention particulière aux récurrences qu'il remarque dans la disparition des pièces du répertoire. Alors qu'il s'attendait à trouver une répartition homogène de ces extinctions au cours du temps, il s'aperçoit que le milieu du XVIII ${ }^{\mathrm{e}}$ siècle et le milieu du XIX ${ }^{\mathrm{e}}$ siècle furent des périodes particulièrement funestes pour de nombreuses œuvres et que le répertoire s'est relativement peu renouvelé durant les années 1790 et la période fin-de-siècle, cent ans plus tard. Bidou s'arrête assez longuement sur les années 1830 et 1850, durant lesquelles disparaissent les pièces de Voltaire, à l'exception de Zaïre, ainsi que plusieurs textes incontournables du répertoire du XVIII $^{\mathrm{e}}$ siècle. Vers 1750 , beaucoup de pièces importantes du XVII ${ }^{\mathrm{e}}$ siècle ont également disparu du répertoire, à l'exception de celles de Molière, de Corneille et de Racine. Bidou ne propose aucune généralisation historique pour tenter d'expliquer ces disparitions en milieu de siècle, mais le fait qu'il ait pu facilement identifier ces tendances indique le genre d'usages nouveaux qu'un tel mode de présentation des données a pu générer. En 1901, Clarétie avait examiné l’histoire des représentations des pièces de onze auteurs parmi les plus joués à l'époque et en avait tiré un récit qui renforçait les stéréotypes nationaux sur la gaieté et le rire français. En 1921, dans le sillage des pertes catastrophiques de la France pendant la Première Guerre mondiale aussi bien en termes démographiques que culturels, Bidou étudie de façon plus rigoureuse les grandes tendances du répertoire sur vingt-quatre décennies. Les nouvelles données et les nouvelles approches statistiques générèrent ainsi de 
nouvelles questions qui allaient résonner au-delà des frontières françaises.

Malheureusement, Joannidès succomba à une maladie cardiaque en 1927, au jeune âge de quarante-sept ans, et il ne put voir comment d'autres prolongèrent son travail $\underline{28}$.

\section{Henry Carrington Lancaster : un membre éminent de l'Académie américaine (1941-1951)}

Le travail de Mouhy au milieu du XVIII ${ }^{\mathrm{e}}$ siècle comme celui de Joannidès au début du $\mathrm{XX}^{\mathrm{e}}$ furent guidés par un engagement direct avec l'histoire en cours de la ComédieFrançaise. Le chercheur américain Henry Carrington Lancaster avait une motivation bien différente lorsqu'il entreprit de transcrire en partie les registres de recettes. Sa compilation en deux parties, The Comédie-Française, 1680-1700. Plays, Actors, Spectators, Finances (1941) et The Comédie-Française, 1701-1774. Plays, Actors, Spectators, Finances (1951), vint accompagner son œuvre monumentale en neuf volumes, qui constitue toujours un ouvrage de référence pour la recherche sur le théâtre français d'Ancien Régime : A History of French Dramatic Literature in the Seventeenth Century (1929-1942)르. Ces études, et plus généralement la majeure partie de la carrière professionnelle de Lancaster, furent influencées par son expérience des deux guerres mondiales, mais aussi par sa position prédominante au sein de l'Académie américaine. Non sans emphase, Lancaster dédicace le dernier tome de sa grande étude en plusieurs volumes à « La Victoire ». Consacré à la scène dramatique du XVII ${ }^{\mathrm{e}}$ siècle, ce tome fut publié en pleine Seconde Guerre mondiale, en 1942. Dans le dernier paragraphe, synthétisant les 3700 pages qui précèdent sur le théâtre entre 1610 et 1700, Lancaster écrit :

Limiter sa connaissance à trois auteurs de premier plan [Corneille, Racine et Molière] est comparable à la vieille méthode consistant à étudier l'Histoire uniquement par le prisme des guerres, des négociations politiques et de la vies privées des rois et des reines. Le triomphe imminent de la démocratie, que ce soit à un niveau militaire ou politique, devrait coïncider avec la volonté des chercheurs à prêter attention à des auteurs mineurs qui viennent en complément des œuvres de plus grands hommes. Avec eux, ils reflètent les aspirations pour les arts et pour la vie d'un siècle où le gouvernement français refusait de céder aux intrusions étrangères et donnait aux auteurs français la possibilité de dramatiser ce qui est, dans le comportement humain, vivifiant ou pathétique, admirable ou risible. $\underline{30}$ 
En d'autres termes, son approche des registres et, de manière plus générale, de l'histoire théâtrale et littéraire, témoigne d'un sens de l'égalité influencé par son époque et par sa culture. Lancaster avait l'ambition d'écrire l'histoire du théâtre au XVII ${ }^{\mathrm{e}}$ siècle en ne s'arrêtant pas aux biographies habituelles du trio canonique ou aux évaluations esthétiques de leurs œuvres. Il défendait l'idée qu'une histoire globale du théâtre de cette période éclairerait celle du pays dans son ensemble et qu'elle permettrait d'ouvrir en retour de nouvelles perspectives critiques sur les dramaturges les plus connus. Compte tenu de ces ambitions scientifiques, il n'est pas surprenant que, des trois auteurs étudiés dans cet article, ce soit Lancaster qui propose les reproductions des registres les plus complètes.

Lancaster est né à Richmond en Virginie en 1882, trois ans après la naissance de Joannidès en Angleterre [Fig. 10].

Il fit ses études universitaires à l'Université de Virginie et reçut son doctorat (Ph. D.) de l'Université Johns Hopkins en 1907. Il obtint son premier poste d'enseignant à Amherst College, dans l'ouest du Massachusetts, où il resta douze ans. Pendant la Première Guerre mondiale, et bien qu'il n'ait pas été éligible au service militaire, il s'engagea volontairement en tant que Directeur américain de l'éducation des Foyers du soldat en France. En 1919, il retourna à Johns Hopkins en tant que professeur et y resta jusqu'à sa mort en 1954. Il dirigea le département de langues et littératures romanes de Johns Hopkins pendant près de trente ans et il fut pendant vingt-six ans l'éditeur de Modern Language Notes, l'une des publications majeures de la Modern Language Association (MLA), qui est la principale société savante aux États-Unis pour les études littéraires et philologiques et dont il fut le président en 1939. Il fut aussi le correspondant de la Revue d'Histoire littéraire de la France. Gustave Lanson et Daniel Mornet connaissaient ses travaux et en firent le compte rendu. En 1932, le gouvernement français le nomma Chevalier de la légion d’honneur et il reçut un grade honorifique de la Sorbonne en 1946쓸. Ainsi, à la différence de Mouhy, qui était un auteur motivé mais sans talent, à la marge des institutions de son temps, et de Joannidès, dont la carrière est étroitement liée au monde parisien des artistes et des intellectuels des années 1890 à 1920, Lancaster était un membre incontournable de l'Académie américaine et jouissait de relations prestigieuses à l'étranger. Pendant la première moitié du $\mathrm{XX}^{\mathrm{e}}$ siècle, il fut l'une des figures les plus importantes dans le champ des études en langues romanes aux États-Unis, à une époque où l'Académie américaine était en train d'acquérir une réputation internationale. 
C'est entre autres grâce à ses nombreuses publications scientifiques que Lancaster acquit une grande autorité institutionnelle au cours de sa carrière. Même si son History of Dramatic Literature in the Seventeenth Century est l'ouvrage à l'influence la plus durable, il a également publié à partir de sa thèse de doctorat une monographie sur la tragi-comédie française entre 1552 et 1628, ainsi qu'une étude sur le dramaturge du XVII ${ }^{\mathrm{e}}$ siècle Pierre du Ryer. La liste de ses publications est également riche des éditions critiques d'une demi-douzaine de pièces, aussi bien d'auteurs célèbres que d'auteurs méconnus du XVII ${ }^{\mathrm{e}}$ siècle, et de douzaines d'articles et de comptes rendus. Après la Seconde Guerre mondiale, il ajouta trois volumes à son histoire du théâtre du XVII ${ }^{\mathrm{e}}$ siècle, qui en comportait déjà neuf, pour s'intéresser au $\mathrm{XVIII}^{\mathrm{e}}$ siècle, en se concentrant principalement sur le genre tragique.

Vers la fin de sa carrière, il fit fond sur sa reconnaissance universitaire pour jouer un rôle public. Au début de la Seconde Guerre mondiale, son capital institutionnel et intellectuel commença à transparaître à travers une série de déclarations publiques liant le travail du chercheur en littérature à la survie des démocraties occidentales. Dans son adresse présidentielle de 1940 pour la Modern Language Association, il décrit la tâche qui attend les membres de l'association comme étant la transmission, génération après génération, d’une tradition savante européenne :

Nous sommes comme le fils ayant hérité de son père l'entreprise familiale. Le jeune homme a été préparé à remplir cette fonction mais demeurent de nombreuses questions qu'il aurait aimé poser et de nombreuses responsabilités auxquelles il réalise devoir faire face seul désormais. L'espoir réside dans le fait que ce jeune homme a peut-être des qualités qui se révèleront au gré de la nécessité et qui lui apporteront des succès plus grands que ceux qu'il aurait connus s'il était resté sous l'égide paternelle. $\underline{32}$

Dans une série d'interventions radiophoniques diffusées à Baltimore en 1940 et en 1941, Lancaster s'adresse à ses « concitoyens américains » et évoque des sujets tels que la lutte à mort entre les alliés et les puissances fascistes, la résistance grecque à Mussolini et les devoirs incombant aux États-Unis et aux citoyens en temps de guerre $\underline{33}$. Il débute son émission de juin 1940 avec cette anecdote :

Le 6 juin, au deuxième jour de la grande guerre, je reçus d'un ami français que je n'avais pas vu depuis quinze ans, le câblogramme suivant, long de cinq mots : «Civilisation en danger de mort ». Il m’avait écrit parce que j'étais le seul 
américain qu'il connaissait, originaire du seul pays qui pourrait sauver la civilisation que lui et nous connaissons et aimons. $\underline{34}$

$\mathrm{Au}$ fond, il fit fructifier sa carrière universitaire pour répondre aux besoins idéologiques d'une nation en guerre. Il continua dans la période d'après-guerre à jouer ce rôle d'intellectuel public. En 1949, quand les opinions anti-communistes maccarthystes commencèrent à se diffuser dans l'Académie, et plus largement dans l'ensemble du pays, il prêta son nom comme plaignant principal à un dossier d'instruction visant à infirmer une loi de « sédition d'urgence » passée par l'assemblée législative du Maryland contre de prétendus infiltrés communistes. Il fut rejoint dans cette entreprise juridique par d'autres professeurs de la région de Baltimore et de Washington D.C., ainsi que par un commerçant, un docteur et un sculpteur. La légion américaine, une corporation locale d'avocats et deux organisations syndicales déposèrent un mémoire «Amici Curiae $\underline{35}$ » en soutien à l'action de Lancaster. Ancien volontaire de la Première Guerre mondiale, fervent partisan de l'effort de guerre pendant la suivante et voix de la raison dans un moment d'hystérie collective au début de la Guerre froide, Lancaster avait des intérêts qui allaient bien au-delà des campus prestigieux de la côte Est et qui en ont fait un acteur à part entière des événements majeurs qui secouèrent son époque.

Les turbulences de la Seconde Guerre mondiale permettent également d'expliquer comment cette éminente figure de l'Académie américaine en est venu à publier, en deux volumes et sur dix années, une partie des registres des recettes de la ComédieFrançaise. Grâce aux doctorants qui travaillaient sous sa direction et qui étaient à Paris dans les années 30, Lancaster savait que la Comédie-Française conservait pour toutes les soirées depuis le dernier quart du XVII ${ }^{\mathrm{e}}$ siècle et jusqu'à la fin du XVIII $^{\mathrm{e}}$ siècle une trace de la fréquentation quotidienne du théâtre. À la fin des années 30, au moment où il s'apprêtait à écrire les deux derniers volumes de son étude magistrale sur la période qui va de la mort de Molière, en 1673, au début du siècle suivant, il chercha un moyen de consulter ces documents d'archives. Quand vint 1938, il savait qu'il ne serait plus possible de voyager à Paris pour examiner ces registres et il demanda donc aux autorités parisiennes des reproductions des registres sur microfilms. Il dut solliciter l'appui de la Bibliothèque du Congrès des États-Unis dans ses négociations. À partir de mai 1939, les microfilms commencèrent à arriver à Washington, au moment même où Lancaster lançait son travail sur ses deux derniers volumes. Bien qu'une seconde livraison arrivât en septembre 1939, il lui manquait toujours les microfilms concernant les années essentielles allant de 1681 à 1688, du 
fait de l'invasion de la Pologne par l'Allemagne. Inquiet, Lancaster se renseigna par l'entremise d'un ami américain. Léditeur parisien de celui-ci découvrit que le bureau de la censure français retenait les microfilms depuis septembre pour une raison inexpliquée. Ils furent finalement transmis en novembre et arrivèrent à Washington en décembre, ce qui permit à Lancaster de les consulter alors qu'il poursuivait ses recherches et achevait d'écrire ses derniers volumes $\underline{36}$.

Presque arrivé à la fin de son histoire du théâtre français du XVII ${ }^{\mathrm{e}}$ siècle, Lancaster eut aussi l'idée de publier une transcription partielle des précieuses données que renfermaient les microfilms. Toujours aiguillonné par cette exigence d'exhaustivité qui caractérise son œuvre scientifique aux proportions épiques, il décida de publier un nombre d'informations excédant largement celles de Mouhy ou de Joannidès. Connaissant bien leurs travaux (il se montrait d'ailleurs très critique à l'égard du premier), il avait bien vu que ses prédécesseurs, d'abord intéressés par l’histoire institutionnelle de la Comédie-Française, n'avaient mentionné qu'une partie des représentations dans leurs tableaux. L'histoire théâtrale proposée par Lancaster se penchait également sur l'aspect économique de la représentation et ne nécessitait pas autant de développements textuels, compte tenu de ses nombreux autres écrits sur le sujet pendant cette période. Par conséquent, il décida de reproduire un extrait des registres qui donnerait pour chaque soirée six types de données : la date, les titres des pièces jouées, le nombre de spectateurs présents, la recette totale, la part revenant à chaque comédien et celle du dramaturge pour les créations. En revanche, il décida qu'une publication intégrale des registres « serait extrêmement coûteuse et ne permettrait pas aux lecteurs d'obtenir les informations qu'ils pourraient rechercher $\underline{37}$ ». Le volume qui paraît en 1951 couvre la période allant de 1700 à la mort de Louis XV en 1774 et constitue la base de ses deux ouvrages sur la tragédie au XVIII ${ }^{\mathrm{e}}$ siècle, ayant aussi pour borne chronologique l'année 1774. Bien que les registres aient été tenus jusqu'à la scission entre royalistes et républicains en 1793 et que Lancaster ait consacré le dernier volume de son histoire du théâtre à la tragédie entre le début du règne de Louis XVI en 1774 et les premières heures de la Révolution, il mourut avant de pouvoir publier la transcription des registres correspondant à la période après 1774. 
598

\begin{tabular}{|c|c|c|c|c|}
\hline & \multicolumn{4}{|c|}{ April } \\
\hline 6 & Phèdre, Cocher supposé & 648 & 1012. 1 & \\
\hline 7 & Tartuffe, Escarbagnas & 505 & 734.13 & \\
\hline 8 & $\begin{array}{l}\text { Andromaque, Florentin } \\
\text { Avare, Mariage forcé }\end{array}$ & 291 & 394. $8 \frac{1}{2}$ & \\
\hline 9 & $\begin{array}{l}\text { Avare, Mariage forcé } \\
\text { Thésée, Eté des coque }\end{array}$ & 528 & $644.12 \frac{1}{2}$ & \\
\hline 10 & $\begin{array}{l}\text { Thésée, Eté des coqu } \\
\text { Menteur, Charivari }\end{array}$ & 486 & 7 & \\
\hline 11 & $\begin{array}{l}\text { Menteur, Charivar } \\
\text { Bajazet, Merlin Dragon }\end{array}$ & $\begin{array}{l}497 \\
333\end{array}$ & 40 & \\
\hline 12 & Misanthrope, Plaideurs & 372 & 489 & \\
\hline 13 & Cinna, Souper mal & & & \\
\hline & apprêt & 397 & 642 & \\
\hline 14 & Coquette, Fragments & 282 & 350. $3 \frac{1}{2}$ & \\
\hline 15 & Rodogune, Deuil & 283 & 361.13 & \\
\hline 16 & Esprit follet, Parisienne & 519 & 677. 5 & \\
\hline 17 & Cid, Sicilien & 554 & 705 & \\
\hline 18 & $\begin{array}{l}\text { Femmes sav., Cocu } \\
\text { imaginaire }\end{array}$ & 370 & 501.10 & \\
\hline 19 & Héraclius, Médecins & 233 & 338.17 & \\
\hline 20 & $\begin{array}{l}\text { Homme à bonne fortune, } \\
\text { Médecin malgré lui }\end{array}$ & 556 & 702. 9 & \\
\hline 21 & Nicomède, George Dandin & 220 & $289.17 \frac{1}{2}$ & \\
\hline 22 & Etourdi, Florentin & 191 & $219.16 \frac{1}{2}$ & \\
\hline 23 & Géta, Scapin & 269 & 411. 9 & \\
\hline 24 & Festin de Pierre, Sicilien & 555 & 650.8 & \\
\hline 25 & Phèdre, Sérénade & 350 & 547.10 & \\
\hline 26 & $\begin{array}{l}\text { Ecole des femmes, Souper } \\
\text { mal ap. }\end{array}$ & 243 & 316. $5 \frac{1}{2}$ & \\
\hline 27 & $\begin{array}{l}\text { Pirame et Thisbé, Cocher } \\
\text { supposé }\end{array}$ & 492 & 772. $5 \frac{1}{2}$ & \\
\hline 28 & Esope, Sérénade & 428 & 513.18 & \\
\hline 29 & Pompée, Escarbagnas & 341 & 497. 2 & \\
\hline 30 & Ecole des maris, Grondeur & 589 & $879.16 \frac{1}{2}$ & \\
\hline \multicolumn{5}{|l|}{ May } \\
\hline 1 & $\begin{array}{l}\text { Andronic, Je vous prends } \\
\text { s. v. }\end{array}$ & 849 & 1129. $8 \frac{1}{2}$ & \\
\hline 2 & $\begin{array}{l}\text { Bourgeoises a la m., Je } \\
\text { vous pr. }\end{array}$ & 402 & 509.14 & \\
\hline 3 & Cinna, Eté des coquettes & 357 & 477.15 & \\
\hline 4 & usicien, Je vous & 561 & 883.1 & 2 \\
\hline 6 & Dragons & 564 & 816 & \\
\hline 7 & $\begin{array}{l}\text { Femmes sav., Je vous } \\
\text { prends }\end{array}$ & 547 & 876 & 22 \\
\hline 8 & Cléopâtre, Parisienne & 621 & 850. 1 & 2 \\
\hline 9 & $\begin{array}{l}\text { Coquette, Je vous prends } \\
\text { s. v. }\end{array}$ & 333 & 420. $9 \frac{1}{2}$ & \\
\hline 10 & $\begin{array}{l}\text { Cléopatre, Esprit de } \\
\text { contradic. }\end{array}$ & 392 & $594.19 \frac{1}{2}$ & 17 \\
\hline 11 & Tartuffe, George Dandin & 558 & 766. 4 & 21 \\
\hline 12 & , Fête de village & 364 & 689. 5 & 12 \\
\hline 13 & Médecins & 226 & $238.11 \frac{1}{2}$ & \\
\hline 14 & Grondeur & 695 & 1131.14 & 33 \\
\hline 30 & Gabinie, Sérénade & 520 & 778. $2 \frac{1}{2}$ & 19 \\
\hline 31 & Esprit follet, Escarbagnas & 137 & 149. 9 & \\
\hline \multicolumn{5}{|l|}{ June } \\
\hline $\begin{array}{l}1 \\
2\end{array}$ & $\begin{array}{l}\text { Cléopatre, Mariage forcé } \\
\text { Esope, Souper mal }\end{array}$ & 253 & 327 & \\
\hline & & 387 & & \\
\hline 3 & , Dragons & 118 & 136. 7 & \\
\hline 4 & Cinna, Trois Gascons & 930 & 1341.6 & 37 \\
\hline 5 & eur, Scapin & 234 & 245. 5 & \\
\hline 6 & Ariane, Trois Gascons & 479 & $667.5 \frac{3}{2}$ & \\
\hline 7 & & 269 & $329.16 \frac{1}{2}$ & \\
\hline
\end{tabular}

Dats

8 Britannicus, Trois Gascons

22 Pompée, Trois Gascons

23 Femmes sav., Crispin médecin

24 Sertorius, Trois Gascons

25 Jodelet prince, Esprit de cont.

26 Thésée, Trois Gascons

Homme à b. fort., Eté des coq.

28 Cléopâtre, Trois Gascons

29 Joueur, Sérénade

30 Cid, Trois Gascons

July

1 Crispin musicien, Médecin

$\begin{array}{clll}\text { m. } 1 . & 279 & 327.14 \frac{1}{2} & 3 \\ 2 \text { Alcibiade, George Dandin } & 211 & 374 & 6\end{array}$

3 Jodelet maitre, Mariage forcé

Nicomède, Vendanges de Suresn.

5 Etourdi, Cocu imaginaire $2600323.12 \quad 4$

6 Bajazet, Cocher supposé $199 \quad 287.18 \frac{1}{2} \quad 2$

7 Mère coquette, Plaideurs $221 \quad 264.71$ 8 Pirame et Thisbé, Souper mal ap.

[TRANS. AMER. PHIL. SOC.

SPEC. Receipts Actors Author

$\begin{array}{rlrl}588 & 850.16 \frac{1}{2} & 19 & 29 \\ 410 & 568.14 \frac{1}{2} & 8 & 16.4 \\ & & & \\ 156 & 174.16 \frac{1}{2} & 0 & \\ 582 & 797.2 & 14 & 28.10 \\ & & & \\ 427 & 577.10 & 13 & \\ 513 & 652.2 \frac{1}{2} & 12 & 20.6 \\ & & & \\ 477 & 699.15 & 17 & \\ 294 & 410.5 & 3 & 7.13 \\ 736 & 927.7 \frac{27}{3} & 27 & \\ 356 & 436.10 & 3 & 9.1 \frac{1}{2}\end{array}$

10 Oreste et Pilade, Moulin de Jay, $238 \quad 264.14$ t

11 Festin de Pierre, Florentin 222 239. $81 \quad 0$

12 Polixène, Deuil $52 \quad 53.3 \frac{1}{4} 0$

13 Misanthrope, Médecins $214 \quad 225,7 \frac{1}{4} \quad 0$

14 Rodogune, Esprit de contradic.

$\begin{array}{lll}\text { Tartufie, Sérénade } & 112 & 146.3 \frac{3}{6} \\ & 203 & 217.5\end{array}$

6 Edipe, Escarbagnas 155 197. 71

17 Joueur, Merlin Dragon 481 535. $7 \frac{1}{2} \quad 0$

18 Manlius, Eté des coquettes 184 261. 71 0

19 Jodelet prince, Cocher $\begin{array}{llll}\text { supposé } & 226 & 289,41 & \end{array}$

20 Horace, Sicilien $\quad 244 \quad 313.12 \frac{21}{2} \quad 2$

21 Avare, Cocu imaginaire $\quad 369 \quad 484.713$

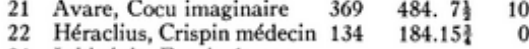

24 Iphigénie, Esprit de contradic. $410 \quad 519.81$

25 Grondeur, Pourceaugnac 545 654. $3 \frac{1}{6} \quad 13$

26 Venceslas, Petit Maitre de $\begin{array}{llllll}\text { campagne } & 417 & 525 & 15 \frac{1}{4} & 11 & 18.12\end{array}$

27 Esprit follet, Médecins

28 Phèdre, Petit Maitre de campag.

$\begin{array}{rrr}417 & 52515 \frac{1}{2} & 11 \\ 114 & 124,3 \frac{1}{8} & 0\end{array}$

29 Ecole des maris, Pourceaugnac

$\begin{array}{llll}220 & 262.12 ? & 0 & 5.14\end{array}$

30 Cléopâtre, Petit Maítre de$$
3 \text { 161. } 3 \quad 0
$$
August

1 Cinna, Florentin $144 \quad$ 222. $2 \frac{1}{2} \quad 0$

2 Femmes savantes,

Femantes, Mariage forcé

3 Mithridate, Deuil

4 Dom Bertrand, Crispin médecin

6 Menteur, Scapin $\begin{array}{lll}1076 & 1702.15 & 52\end{array}$

255 319. 9\} 3

644 1102. $6 \quad 35$ $\begin{array}{lll}101 & 107.12 & 0\end{array}$

Figure 11. Henry Carrington Lancaster, The Comédie-Française, 1701-1774. Plays, Actors, Spectators, Finances, Philadelphia, American Philosophical Society, 1951, Qu'a donc réalisé Lancaster ? La premièje ggggse que l'on pourrait remarquer en étudiant une page du volume de 1951, c'est que sa présentation est plus 
rigoureusement tabulaire que celle de Mouhy [Fig. 11]. Lancaster n'ajoute aucune donnée qui ne se trouve pas dans les registres, à la différence de l'ouvrage de son prédécesseur qui fournit de brefs résumés des pièces. En outre, le travail de Lancaster ressemble davantage à un « calendrier » qu'à un «dictionnaire » : les informations concernant 94 saisons sont présentées de manière chronologique en deux volumes. La seule trace de classement alphabétique se trouve dans les index des pièces et des personnes, incluant acteurs et dramaturges. Par rapport à Mouhy ou Joannidès, Lancaster essaie de reproduire plus fidèlement les registres pour les soumettre à l'examen des lecteurs. Il utilise les possibilités offertes par la page imprimée pour présenter un compte rendu exact des données que contiennent les registres mais, à la différence de Joannidès, il laisse au lecteur le soin de se livrer à toute sorte de calculs.

Les catégories que Lancaster dégage à partir des registres indique qu'il cherche à proposer des informations sur la Comédie-Française en tant qu'institution économique ainsi qu'à fournir des données permettant de réfléchir à la popularité des pièces et des auteurs. Lancaster s'était d'ailleurs penché sur ces deux questions - la stabilité économique de la troupe et la popularité des pièces - dans son histoire du théâtre français. Sa transcription partielle des registres donne donc accès à certaines des sources sur lesquelles il s'est lui-même appuyé. Il concevait clairement son lectorat comme composé d'universitaires aussi bien francophones qu'anglophones : la préface, l'introduction et les notes sont toutes en anglais, permettant aux spécialistes de littérature et aux historiens ne parlant pas français d'utiliser les transcriptions de Lancaster. À la différence de Mouhy, qui avait un but commercial, ou de Joannidès, dont les travaux furent publiés en éditions limitées pour les élites culturelles parisiennes du tournant du siècle, les deux volumes de Lancaster furent publiés par The Johns Hopkins University Press et par la Société Américaine de Philosophie dans ses Transactions.

À partir du milieu du XX $\mathrm{XX}^{\mathrm{e}}$ siècle, le capital culturel du Paris du XVIII ${ }^{\mathrm{e}}$ siècle était internationalement établi et la ville était devenu un objet digne d'intérêt pour des chercheurs comme Lancaster désireux d'établir l'héritage culturel des États-Unis d'après-guerre. Grâce à son travail, une version partielle des données contenues dans les registres de la Comédie-Française, arrivés in extremis de Paris à la veille de la Seconde Guerre mondiale grâce à une technologie nouvelle d'imagerie, était désormais disponible en version imprimée à quiconque avait accès à une bonne bibliothèque universitaire. Dans sa recension critique de The Comédie-Française, 1701 1774, publiée dans la Revue d’Histoire Littéraire de la France en 1954, Jacques 
Scherer note la portée de ces données qui dépassaient dorénavant les frontières de l'hexagone :

On ne peut que se féliciter de cette initiative américaine. On ne peut s'empêcher de regretter en même temps que l'état des études d'histoire du théâtre en France soit tel qu'un ouvrage de fond de cette importance, traitant de ce qu'on est convenu d'appeler une de nos gloires nationales, n'ait pu être réalisé par des Français. $\underline{38}$

Le commentaire de Scherer souligne les changements advenus pendant le demi-siècle qui sépare les travaux de Lancaster des premiers résumés publiés par Joannidès à partir des registres. Quand ceux-ci parurent, les contemporains louèrent la manière dont ils liaient l'histoire de la Comédie-Française au patriotisme culturel français. Cinquante ans plus tard, l'importance scientifique des données avait dépassé cette approche nationaliste. La plainte exprimée par Scherer au sujet de l'état de l'histoire du théâtre dans la France d'après-guerre est en même temps l'affirmation que l'histoire de la principale institution théâtrale française au XVIII ${ }^{\mathrm{e}}$ siècle appartenait dorénavant à tous les chercheurs qui s'y intéressaient de par le monde.

\section{Conclusion : la mise en ligne des registres au $X X I^{e}$ siècle}

Ce parcours à travers trois époques durant lesquelles un plumitif, un érudit et un historien de la littérature américain se sont intéressés aux données que contiennent les registres des recettes de la Comédie-Française permet de mieux comprendre le projet actuel de mise en valeur de ces registres et le travail des auteurs du présent volume qui en explorent les usages possibles. La compilation que réalise Mouhy au $\mathrm{XVIII}^{\mathrm{e}}$ siècle est publié à une période où le public de théâtre commence à se considérer comme faisant partie de l'histoire de la scène française et désire de ce fait en savoir plus sur son passé. Un siècle et demi plus tard, les ouvrages de Joannidès continuent à satisfaire les attentes de spectateurs avant tout intéressés par l'histoire et cherchant à se situer dans la longue tradition du théâtre national qui s'étend alors sur plus de deux cents ans et qui s'incarne dans cette troupe du théâtre-musée connu sous le nom de Comédie-Française. Enfin, le travail de Lancaster est plus nettement scientifique et moins patriotique : il se concentre sur des données vieilles de deux siècles afin de répondre aux besoins des chercheurs plutôt qu'à ceux de la troupe, du patriotisme français ou des spectateurs contemporains. En même temps, cette entreprise vise à rassembler les données concernant la Comédie-Française et son 
histoire pour servir un projet politique et culturel qui atteint son paroxysme après 1945 : celui d'une « Civilisation occidentale » conçue comme allant de concert avec les déclarations politiques et économiques des États-Unis et de ses alliés internationaux après la Seconde Guerre mondiale.

Compte tenu de ce contexte, comment interpréter la présentation, au $\mathrm{XXI}^{\mathrm{e}}$ siècle, de ces données tirées des registres et ayant inspiré le présent ouvrage ? La nouveauté la plus évidente qu'apporte le Projet $\underline{\mathrm{RCF}}$ comparé à ses prédécesseurs est d'ordre technologique. L'imprimé était la technique sur laquelle reposaient les travaux de Mouhy, de Joannidès et de Lancaster. Au fil des trois générations qui nous séparent de la première édition des transcriptions partielles des données de 1701 à 1774, de nouveaux médias numériques et des technologies inédites ont ouvert des possibilités originales à un nombre toujours plus grand de chercheurs et de spectateurs. Ces transformations se comprennent peut-être de la manière la plus claire en comparant quelques outils de recherche et de visualisation conçus par l'équipe du Projet RCF aux formats utilisés par Mouhy, par Joannidès et par Lancaster. On pourrait par exemple mettre en regard les tableaux que l'on trouve dans les Tablettes de Mouhy et l'Outil de découverte réalisé par Adrien Di Mascio de l'équipe du Projet RCF. Le principe d'organisation des tableaux dans l'ouvrage de 1752 publié par Mouhy repose sur la liste alphabétique des titres de pièce (voir Figures 2 et 3 ). Une fois que les lecteurs ont trouvé la pièce qui les intéresse, ils peuvent facilement obtenir des informations sur son auteur, ses représentations et son édition. L'Outil de découverte permet aux utilisateurs d'accéder aux mêmes types d'informations à partir du titre de la pièce mais aussi d'explorer la base de données en fonction du nom d'auteur, du genre ou de la saison théâtrale, autant de possibilités que l'on retrouve ci-dessous [Fig. 12].

Visit the web version of this article to view interactive content.

Figure 12. Outil de découverte, Projet RCF.

Cliquer sur « Pièces » pour obtenir la fréquence de représentations des pièces répertoriées dans la base de données et leurs recettes chaque soirée.

On s’aperçoit ainsi que le Projet RCF tire parti des technologies actuelles pour offrir aux lecteurs curieux un accès aux données historiques, vérifiées et mises à jour de manière rigoureuse, sur les auteurs, les pièces, les genres et les saisons, ainsi que des 
visualisations grâce auxquelles on peut repérer plus facilement des tendances dans les représentations au fil des siècles.

De manière analogue, on pourrait comparer les compilations de données que fait

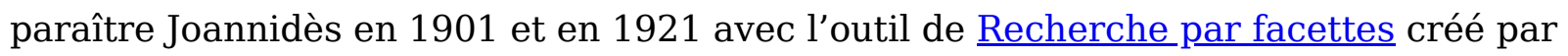
Christopher York et modifié par une équipe de l’Université Laval au Canada. Dans ses deux ouvrages, Joannidès est parvenu à transformer les données brutes des registres en une série de tableaux synthétisant les fréquences auxquelles pièces et dramaturges sont représentés tout au long des 240 saisons théâtrales. Il propose aussi des informations pour des saisons en particulier, pour toute la carrière d'un auteur et pour chaque pièce encore au répertoire de la troupe au moment de la publication (voir Figures 5-9). Comme on peut le voir ci-dessous, l'outil de Recherche par facettes permet également aux utilisateurs d'étudier la fréquence des représentations d'auteurs ou de pièces, soit sur une seule année, soit tout au long des 113 saisons que répertorie la base de données du Projet RCF [Fig. 13].

\section{Visit the web version of this article to view interactive content.}

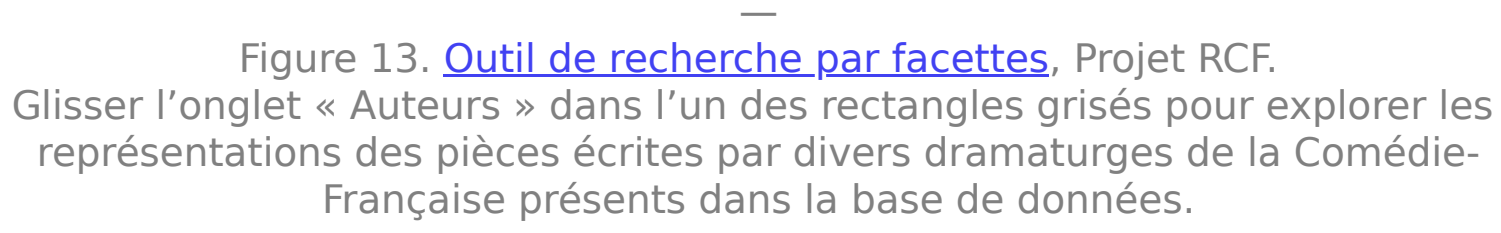

De plus, l'outil de Recherche par facettes calcule le total des recettes et les revenus générés par le seul parterre au cours de la carrière d'un auteur ou d'une pièce au répertoire de la Comédie-Française jusqu'à 1793. Il propose également une synthèse des recettes pour les représentations quotidiennes et permet aux utilisateurs d'accéder à une fiche qui présente quelques données inscrites sur des pages des registres. Ainsi, le Projet RCF réalise en ligne le même travail que celui de Joannidès, auquel il ajoute cependant la possibilité d'accéder aux données relatives aux ventes et aux entrées, là où son précurseur se limitait aux fréquences des représentations.

Enfin, la reproduction en ligne des registres des recettes quotidiennes permet de les compulser dans leur totalité et constitue un équivalent numérique des transcriptions partielles que Lancaster a publié en 1941 puis en 1951. Lancaster avait estimé que donner le détail des ventes en fonction des prix des places n'intéresserait pas 
suffisamment ses lecteurs et il avait donc choisi de ne pas les inclure. En revanche, la nature numérique du Projet RCF permet de reproduire en haute définition les registres originaux sans qu'il ne soit nécessaire de laisser de côté certaines catégories de données. La comparaison de la reproduction intégrale du registre de la saison 17011702 ci-dessous [Fig. 14] avec le nombre limité d’informations économiques que propose l'ouvrage de Lancaster en 1951 [Fig. 11] fait clairement apparaître cela.

\section{Visit the web version of this article to view interactive content.}

\section{Figure 14. Registre R46, Saison 1701-02. Flipbook du Projet RCF.} Cliquer sur l'une des deux pages ou déplacer le curseur en bas de la page pour « tourner » les pages et voir les informations concernant d'autres représentations de la saison.

Si Lancaster ne se trompait pas en pensant que l'imprimé au milieu du $\mathrm{XX}^{\mathrm{e}}$ siècle ne disposait ni du temps ni de l'argent nécessaires pour reproduire l'ensemble des informations contenues dans les pages de chaque registre, les technologies numériques actuelles offrent davantage de possibilités éditoriales. En outre, notre dernier outil de recherche et de visualisation, le Tableau croisé dynamique (également conçu par Christopher York et optimisé par l'équipe de l'Université Laval), permet aux utilisateurs d'examiner dans les moindres détails la répartition des représentations des pièces et des dramaturges, ainsi que leurs recettes, par mois, par année ou par décennie. Il génère des graphiques linéaires et des diagrammes à barres permettant aux utilisateurs d'identifier des récurrences dans les données que l’on peut ainsi explorer avec plus de précision. Il présente également sous la forme d'un calendrier des informations sur les fréquences et les revenus suggérant des variations à l'échelle de la semaine et de la saison sur les 113 années de données disponibles [Fig. 15].

\section{Visit the web version of this article to view interactive content.}

Figure 15. Tableau croisé dynamique, Projet RCF.

Utiliser le menu déroulant sur la gauche pour changer les critères pris en compte dans le tableau et le graphique linéaire sur la droite.

Le but de ces comparaisons n'est bien entendu pas de dénigrer les ouvrages et la créativité de ceux qui ont publié, sous une forme ou une autre, les données contenues 
dans les registres de la Comédie-Française avant l'existence du Projet $\underline{\mathrm{RCF}}$. Mouhy, Joannidès et Lancaster ont tous travaillé à un moment différent, avec des supports et des technologies différents et pour des lecteurs ayant des attentes qui ne sont pas celles d'aujourd'hui. Chacun d'eux a pu apprendre de son prédécesseur, de la même manière que notre vision du Projet $\underline{\mathrm{RCF}}$ s'est développée en prenant en compte les succès et les limites des travaux de ceux qui sont venus avant nous. Il est important de signaler que leurs études perdurent sous forme de livres disponibles dans les bibliothèques physiques, dont l'existence permet de conserver le riche héritage livresque du passé. Les technologies numériques d'aujourd'hui offrent un accès autrefois inimaginable aux registres, vingt-quatre heure sur vingt-quatre et sept jours sur sept, partout dans le monde. Toutefois, notre confiance en la pérennité de ces technologies sur le long terme et en notre détermination à créer des plateformes de conservation qui les préserveront pour les générations à venir est pour le moins incertaine. Comme nous le signalions dans l'introduction de cet ouvrage, il n'est pas possible d'être sûr que ces mots seront encore accessibles en ligne sous ce format d'ici cinq ans.

Même si ces questions de préservation devraient à juste titre nous faire réfléchir, le contexte technologique, politique et culturel actuel profite au Projet $\underline{\mathrm{RCF}}$ au moins de deux façons. Tout d'abord, les relations entre la communauté scientifique et un public composé de curieux et d'amateurs sont peut-être plus étroites que ne le pense la première, puisque les évolutions des normes de présentation qui ont cours sur internet ont nécessairement influencé la conception du Projet $\underline{\mathrm{RCF}}$. Quiconque ayant à disposition un appareil connecté peut parcourir les données et concevoir des hypothèses de recherche. En retour, cette accessibilité mondiale et illimitée lance un pont entre des questionnements scientifiques ciblés et un public plus large pouvant faire un usage divers de ces données $\underline{39}$. En second lieu, la riche tradition scientifique n'est pas rendue obsolète par les analyses de données que permet le Projet $\underline{\mathrm{RCF}}$. Bien peu de chercheurs en sciences humaines choisiront de délaisser les explications de texte et les interprétations dramaturgiques pour se contenter de faire défiler des pages internet et de cliquer sur des liens. La plupart d'entre eux seront intéressés par ce que peuvent apporter aux débats déjà riches dans nos disciplines les analyses visuelles d'immenses quantités de données. Dans le cas du Projet $\underline{\mathrm{RCF}}$, les données concernant les recettes générées par les entrées pour chaque soirée participe à l'élargissement général des perspectives dans les études sur le théâtre et la représentation. L'analyse littéraire des textes dramatiques est désormais complétée par l'attention prêtée aux conditions matérielles et économiques. En résulte une 
compréhension plus complète du passé théâtral, prenant en compte les usages politiques, sociaux et culturels de la scène aussi bien que l'esthétique des textes et de leurs mises en scène $\underline{40}$. Tout en explorant le site du Projet $\underline{\mathrm{RCF}}$, on pourrait imaginer Mouhy, Joannidès et Lancaster aujourd'hui en train de jouer avec les données des registres sur leur tablette et se demander quelles conversations ils auraient pu avoir, entre eux ou avec nous, au sujet du premier répertoire de la Comédie-Française.

\section{Footnotes}

1. Daniel Allington, Sarah Brouillette et David Golumbia, « Neo-Liberal Tools (and Archives) : A Political History of Digital Humanities », The Los Angeles Review of Books, 1er mai 2016, https://lareviewofbooks.org/article/neoliberaltools-archives-political-history-digital-humanities/. Ce texte polémique a suscité de nombreuses critiques dans les jours et les semaines qui ont suivi sa parution. Un échantillon de ces critiques est disponible ici : https://digitalhumanitiesnow.org/2016/05/editors-choice-a-second-round-up-ofresponses-to-the-larb-critique/.

2. Timothy Brennan, «The Digital-Humanities Bust », The Chronicle of Higher Education, 15 octobre 2017, https://www.chronicle.com/article/The-DigitalHumanities-Bust/241424. Une réponse fut publiée la semaine suivante : Sarah E. Bond, Hoyt Long et Ted Underwood, "Digital" is not the Opposite of "Humanities" », The Chronicle of Higher Education, 23 octobre 2017, https://www.chronicle.com/article/Digital-Is-Not-the/241634. Une synthèse des réactions publiées sur Twitter est disponible sur le blog Digital Humanities Now (https://digitalhumanitiesnow.org/2017/10/editors-choice-twitters-response-to-thedigital-humanities-bust/). $\subseteq$

3. Charles de Fieux, Chevalier de Mouhy, Tablettes dramatiques, contenant l'abrégé de l'histoire du théâtre françois, l'établissement des théâtres à Paris, un dictionnaire des pièces, et l'abrégé des auteurs \& des acteurs, Paris, Jorry, 1752. $\Xi$

4. Pour un résumé des écrits sur l'histoire du théâtre français au XVIII ${ }^{\mathrm{e}}$ siècle, voir Martine de Rougemont, La Vie théâtrale en France au XVIII e siècle, Paris, Champion, 1988, p. 84-87.

5. Pierre-François Godard de Beauchamps, Recherches sur les théâtres de France, depuis l'année onze cens soixante-un, jusques à présent, Paris, Prault 
père, 1735. Le troisième et dernier volume propose une série de critiques de spectacles donnés durant les années 1730 à Paris et s'achève par une liste alphabétique des auteurs et des pièces constitutives de la tradition théâtrale française s'étalant sur plusieurs siècles. Léditeur de cet ouvrage, Prault père, fit paraître de façon concomitante en 1735 une édition in-quarto en un seul volume donnant le même texte, augmenté de deux figures synthétisant visuellement l'histoire des salles de spectacle parisiennes et des dramaturges de langue française depuis le Moyen Âge. $ヒ$

6. Claude et François Parfaict, Histoire générale du théâtre françois depuis son origine jusqu'à présent, Amsterdam, 1734-1749, 15 vol. Grâce à cette approche, les frères Parfaict semblent avoir reçu les applaudissements de leurs contemporains ; un critique, écrivant en mars 1748 dans le Journal de Trévoux (officiellement les Mémoires pour l'histoire des sciences et des beaux arts), remarque que le volume 10, qui couvre la période essentielle allant de 1666 à 1669 durant laquelle furent lancées quelques-unes des plus importantes pièces de Corneille, de Molière et de Racine, dessine un portrait idéal de la scène française « au comble de sa gloire » (Mémoires pour l'histoire des sciences et des beaux arts, mars 1748, p. 389).

7. Sur la vie et l'œuvre de Mouhy, voir Annie Rivara, «Un écrivain caméléon, Chevalier inexistant ou figure "d'auteur" hardie ? Lecture et création chez le Chevalier de Mouhy », dans Jan Herman, Kris Peters et Paul Pelckmans (dir.), Le Chevalier de Mouhy. Bagarre et bigarrure, Amsterdam et New York, Rodopi, 2010, p. 7-24 ; Paul d'Estrée, « Un journaliste policier : le Chevalier de Mouhy », Revue d'Histoire Littéraire de la France, $\mathrm{n}^{\circ} 4-2$, 1897, p. 195-238 ; Lawrence Lynch et Patricia Clancy, « Charles de Fieux de Mouhy (1701-1784) », dans Jean Sgard (dir.), Dictionnaire des journalistes, édition électronique revue, corrigée et augmentée, consulté le 5 mai 2020. Sur la longévité de la Médée de Longepierre dans le répertoire de la Comédie-Française, voir https://www.cfregisters.org $L$. 8. La majeure partie des essais qui figurent dans Le Chevalier de Mouhy. Bagarre et bigarrure, op. cit., commentent la production romanesque de Mouhy. $\leftrightarrows$ 9. Robert Darnton fut le premier à identifier une rue parisienne équivalente à la Grub Street londonienne et ses résidents du XVIII ${ }^{\mathrm{e}}$ siècle dans son essai de référence « The High Enlightenment and the Low Life of Literature in Prerevolutionary France », Past and Present, n 51, mai 1971, p. 81-115. Au sujet de débats engendré par le terme de « grub street » et les positions de Darnton 
sur les «petits » de la littérature française en général, voir les essais de Jeremy D. Popkin, Daniel Gordon, Elizabeth L. Eisenstein, David A. Bell et Thomas Kaiser, ainsi que la réponse de R. Darnton dans Haydn T. Mason (dir.), The Darnton Debate. Books and Revolution in the Eighteenth Century, Studies on Voltaire and the Eighteenth Century, n³59, 1998 ; et David A. Bell, «A Grub Street Hack Goes to War », dans Charles Walton (dir.), Into Print. Limits and Legacies of the Enlightenment. Essays in Honor of Robert Darnton, University Park (PA), Penn State UP, 2011, p. 131-144.

10. Les historiens ultérieurs du théâtre ont largement critiqué les erreurs factuelles de Mouhy et sa négligence générale. Henry Carrington Lancaster, sur lequel je reviendrai, condamne le travail de Mouhy dans son examen des histoires du XVIII ${ }^{\mathrm{e}}$ siècle portant sur la scène française du XVII ${ }^{\mathrm{e}}$ siècle, le qualifiant de « pire qu'inutile » (A History of French Dramatic Literature in the Seventeenth Century. Part 1 : The Pre-Classical Period, 1610-1634, Baltimore, Johns Hopkins UP, 1929, p. 5). Je ne me préoccupe pas ici de la précision du travail de Mouhy mais de la nouveauté propre à la présentation des données sous forme tabulaire. $\doteq$

11. Pour plus de détails sur la pièce de Dancourt et la cause célèbre qui l'inspira, voir Jeffrey S. Ravel, The Would-Be Commoner. A Tale of Deception, Murder, and Justice in Seventeenth-Century France, Boston, Houghton Mifflin, 2008. $ヒ$ 12. Charles de Fieux, Chevalier de Mouhy, Abrégé de l'histoire du théâtre françois, depuis son origine jusqu'au premier juin de l'année 1780, Paris, Jorry, 1780, t. I, p. $502 . \uplus$

13. Élie-Catherine Fréron, Lettres sur quelques écrits de ce temps, 1752, vol. 6, Lettre III, p. 43-54.

14. Le premier almanach spécifiquement dévolu au théâtre parisien semble avoir été le Calendrier historique des théâtres de l'Opéra, et des comédies françoises et italiennes et des foires, Paris, Cailleau, 1751. L'année suivante, Duchesne récupéra le titre, qui continua de paraître jusqu'à la fin de l'Ancien Régime. $\leftrightarrows$

15. Calendrier pour l'année MDCCLII à l'usage des comédiens François ordinaires du roi, Paris, Jorry, 1752. $\_$

16. Le Répertoire de toutes les pièces restées au Théâtre François, avec la date, le nombre des représentations, et les noms des Auteurs et des Acteurs vivans, 
Paris, Pissot, Jorry \& Duchesne, 1753.

17. Sur l'histoire du théâtre du XIX ${ }^{\mathrm{e}}$ siècle à Paris, voir F. W. J. Hemmings,

Theatre and State in France, 1760-1905, Cambridge (UK), Cambridge UP, 1994 ;

Sally Debra Charnow, Theatre, Politics, and Markets in fin-de-siècle Paris.

Staging Modernity, New York, Palgrave Macmillan, 2005 ; Jean-Claude Yon, Une

Histoire du théâtre à Paris de la Révolution à la Grande Guerre, Paris, Aubier, 2012 ; Hélène Laplace-Claverie, Sylvain Ledda et Florence Naugrette (dir.), Le

Théâtre français du XIX siècle : histoire, textes choisis, mises en scène, Boynes, L'Avant-scène théâtre, 2008. Sur la concurrence entre les médias et les divertissements à la fin du XIX ${ }^{\mathrm{e}}$ siècle, voir Vanessa Schwartz, Spectacular Realities. Early Mass Culture in fin-de-siècle France, Berkeley and London, University of California Press, 1998.

18. La citation est empruntée à la critique que publia Gustave Larroumet dans Le Temps du 17 mars 1902 au sujet de l'ouvrage de Joannidès, La ComédieFrançaise, 1902 (coupure trouvée à la BnF, Arts du spectacle, cote LJ W-332, "Articles de presse concernant la parution de l'ouvrage d'Alexandre Joannidès, $L a$ Comédie-Française"). Jacqueline Razgonnikoff, dans La Comédie-Française. Le théâtre de la rue de Richelieu de 1799 à nos jours, Paris, Artlys, 2013, p. 43-152, propose une description évocatrice de l'histoire de la Comédie-Française au $\mathrm{XIX}^{\mathrm{e}}$ siècle, fondée sur un examen minutieux des archives de la troupe. $\subseteq$ 19. Sur la vie de Joannidès, voir Jules Clarétie, "Préface », dans Alexandre Joannidès, La Comédie-Française de 1680 à 1900, Paris, Plon, 1901, p. i-vii ; et la notice nécrologique intitulée « Alexandre Joannidès, historiographe de la Comédie- Française (1879-1926) », dans le dossier à son nom que conserve la Bibliothèque-Musée de la Comédie-Française. $\_$

20. Ce récit quelque peu coloré de la manière dont Joannidès découvrit les archives de la Comédie-Française peut être trouvé dans la préface de Jules Clarétie à Alexandre Joannidès, La Comédie-Française de 1680 à 1900, op. cit., p. i-ii. $\subseteq$

21. Jules Clarétie, « Préface », op. cit., p. vii. $\subseteq$

22. Alexandre Joannidès, La Comédie-Française, 1902, avec une préface de J. Truffier, sociétaire de la Comédie-Française, Paris, Plon, 1903, p. i. $\triangleq$ 
23. La postérité du répertoire voltairien à la Comédie-Française est analysée par Pierre Frantz, «Le Moment Voltaire » et Lauren R. Clay, « L'étrange carrière de Voltaire, le dramaturge le plus rentable du XVIII siècle », dans le présent volume.



24. Alexandre Joannidès, La Comédie-Française, 1902..., op. cit., p. i. $\triangleq$ 25. Sur l'autorité grandissante du recours aux nombres dans l'argumentation à la fin du XIX ${ }^{\mathrm{e}}$ siècle, voir Joshua Cole, The Power of Large Numbers. Population, Politics, and Gender in Nineteenth-Century France, Ithaca, Cornell UP, 2000. 26. Jules Clarétie, «Préface », op. cit., p. v. Pour une analyse statistique analogue à la même époque, voir la critique du volume de Joannidès de 1901 par François Brunetière dans La Revue des deux mondes, n 71, 1901, t. 4, p. 954958. $\subseteq$

27. Journal des débats, 14 mars 1921 (coupure trouvée à la BnF, Arts du spectacle, cote LJ W-332, Articles de presse concernant la parution de l'ouvrage d'Alexandre Joannidès). \pm

28. Après avoir fait paraître l'ouvrage en 1921 avec les mises à jour statistiques du répertoire historique de la compagnie, Joannidès publia une synthèse supplémentaire des activités de la troupe en 1925, couvrant les évolutions de la première moitié des années 1920. Il mourut en 1927 au jeune âge de quarantesept ans mais la pratique consistant à faire paraître un annuaire annuel se poursuivit jusqu'à la Seconde Guerre mondiale. $\subseteq$

29. Henry Carrington Lancaster, The Comédie-Française, 1680-1 700. Plays, Actors, Spectators, Finances, Baltimore, Johns Hopkins UP, 1941 ; The ComédieFrançaise, 1701-1774. Plays, Actors, Spectators, Finances, Philadelphie, American Philosophical Society, 1951 ; A History of French Dramatic Literature in the Seventeenth Century, Baltimore, Johns Hopkins UP, 1929-1942, 9 vol. $ヒ$ 30. Henry Carrington Lancaster, A History of French Dramatic Literature in the Seventeenth Century. Part V : Recapitulation, 1610-1700, Baltimore, Johns Hopkins UP, 1942, p. 148.

31. Louis Cons fait l'ébauche de la vie de Lancaster et de ses succès dans son Introduction à Adventures of a Literary Historian. A Collection of His Writings Presented to H.C. Lancaster, Baltimore, The Johns Hopkins UP, 1942, p. vii-xi. $\doteq$ 
32. Henry Carrington Lancaster, « Nineteen-Forty », dans Adventures of a Literary Historian, op. cit., p. 3. $ヒ$

33. Henry Carrigton Lancaster, «In Danger of Death », « Help the Greeks » et «America's Duty », dans Adventures of a Literary Historian, op. cit., p. 56-63. 34. Henry Carrington Lancaster, «In Danger of Death », art. cit., p. 56. 35. Pour un résumé de cette controverse, voir Vernon L. Pederson, The Communist Party in Maryland, 1919-1957, Urbana et Chicago, University of Illinois Press, 2001, p. 166-170 ; et M. J. Heale, McCarthy's Americans: Red Scare Politics in State and Nation, 1935-1965, Athènes, University of Georgia Press, 1998, p. 60-67. NdT. En droit américain, un amicus curiae est une personne ou un groupe de personnes portant à l'attention d'un tribunal des informations au sujet d'une affaire dont elles ne sont pas partie prenante, sous la forme d'un mémoire par exemple. $\triangleq$

36. Les microfilms sont toujours détenus par la Bibliothèque du Congrès des États-Unis où l'équipe du Projet RCF les consulta au début du projet en 2008. À la pointe de la technologie en 1939, ils étaient dans un état de détérioration notable au début des années 2000 et étaient devenus obsolètes du fait de l'avancée des techniques d'imagerie numérique. $\subseteq$ 37. Henry Carrington Lancaster, The Comédie-Française, 1701-1774, op. cit., p. 593. Lancaster exprime une opinion analogue dans The Comédie-Française, 1680-1700, op. cit., p. 7. $\subseteq$

38. Jacques Scherer, Recension critique de Henry Carrington Lancaster, The Comédie-Française, 1701-1774. Plays, Actors, Spectators, Finances, dans Revue d'Histoire Littéraire de la France, n 54-1, janvier-mars 1954, p. 103. 39. Sur les usages imprévus du Projet RCF par les usagers en ligne, voir Jeffrey S. Ravel, « The Comédie-Française Registers Project : Questions of Audience », dans Simon Burrows et Glenn Roe, eds. Digitizing Enlightenment: Digital Humanities and the Transformation of Eighteenth-Century Studies (Oxford Studies in the Enlightenment 2020:7), p. 133-50. Sur les usages en cours du Projet RCF, voir https://www.cfregisters.org/fr/espace-pédagogique? $\mathrm{q}=\mathrm{en} / \mathrm{teachin} \mathrm{g}$-resources et https://www.cfregisters.org/fr/archives? $\mathrm{q} \equiv \mathrm{en} / \mathrm{archives}$. Voir également Christian Biet, « Conversation avec Clément Hervieu-Léger », Littératures classiques, n 95, 2018, p. 35-37, pour les 
considérations d'un metteur en scène actuel de la Comédie-Française sur l'impact que le Projet RCF a pu avoir sur sa compréhension du répertoire de la troupe. $\triangleq$

40. Jeffrey S. Ravel, « Des définitions aux usages. L'historiographie du théâtre français au XVIII ${ }^{\mathrm{e}}$ siècle », Parlement(s). Revue d'Histoire et Politique, H.S. $\mathrm{n}^{\circ}$ 8, 2012, p. 39-52. 